\title{
A FRACTIONAL MULTI-STATES MODEL FOR INSURANCE
}

Donatien Hainaut

\section{LIDAM Discussion Paper ISBA $2021 / 19$}




\title{
A fractional multi-states model for insurance
}

\author{
Donatien Hainaut
}

January 8, 2021

\begin{abstract}
A common approach for pricing insurance contracts consists to represent the insured's health status by a Markov chain. This article extends this framework by observing this chain on a random scale of time, defined as the inverse of an $\alpha$-stable process. This stochastic clock induces sub-exponential waiting times spent in each state. We first review and extend the properties of this time-change to a conditional filtration at time $t>0$. Next we evaluate a general type of insurance contract from inception to expiry.
\end{abstract}

\section{Introduction}

Insurances provide various coverages for all of life's mishaps as disability, unemployment, illness or death. A standard approach for modeling an insurance contract consists to represent the insured's health status by a Markov chain. In this framework, benefits and premiums are contingent upon the state of this chain. Benefits may be lump sums paid at transitions of states or annuities.

Markov chain models have a high level of analytical tractability but some of their properties limit their field of applications. For instance, the number of transitions between two states is a Poisson process with a variance growing linearly with time. For many natural phenomena, this assumption is unrealistic. Musson and al. (2002) point out that earthquake arrival processes have a variance increasing in time in accordance with a power law $t^{\gamma}(\gamma \neq 1)$. Kumar et al. (2020) arrive to similar conclusions in a study of inter-arrival times of claims caused by fire, recorded in a Danish dataset. A way to circumvent this drawback consists to work with non-homogenous semiMarkov processes as in Janssen and De Dominicis (1984), Janssen et Manca (1997) or D'Amico et al. (2009). An alternative is offered by fractional processes since their variance growths as a power law. We observe a growing interest in applications of these processes to insurance from the actuarial community. For instance, Kumar et al. (2020) or Biard and Saussereau (2018) examine the properties of fractional risk insurance processes and their long-range dependence.

On the other hand, the inter-arrival times of Markov chain transitions are exponential random variables. This distribution is memoryless, i.e. the distribution of the waiting time until a certain event does not depend on how much time has elapsed already. In life or healthcare insurance, this feature is not observed in many circumstances. For instance, the disability durations often display a sub-exponential instead of a exponential distribution. This point is emphasized by Zadeh et al. (2014) who propose to model the duration of disability with a phase-type distribution. For this purpose, they add artificial states of decreasing vitality up to death. Albrecher et al. (2020) instead consider a chain with inter-arrival times that are Mittag Leffler distributed. They show that in presence of an absorbing state, the time needed to reach this state has a Matrix Mittag Leffler distribution. In this article, we instead consider a time-changed version of a Markov chain, establish the convergence to the model of Albrecher et al. (2020) and present several new features using fractional calculus.

In this article, the insured's health status is modeled by a Markov chain observed on a random scale of time. The stochastic clock is the inverse of an $\alpha$-stable Lévy process. This clock, also called subordinators, has for particularity to allow for periods during which the time stops. The chain being trapped in a state when the clock is stopped, the distribution of the time of stay is 
sub-exponential. The properties of inverse stable subordinators are studied in Meerschaert and Straka (2013). This time-changed may be combined with Brownian motions (Piryatinska et al., 2005, Magdziarz, 2009), jump-diffusions and shot noise processes (Hainaut, 2020 and Hainaut et Leonenko, 2021), or with Ornstein-Uhlenbeck processes (Leonenko et al., 2013). Beghin and Orsingher (2009), Aletti et al. (2018) investigates the properties of fractional Poisson processes.

Inverse stable subordinators present a major drawback: the time-changed processes are neither Markov nor semi-martingales. As consequence, most of their properties hold only at the birth of the process. Conditionally to information at a later time, subordinated processes lose most of their analytical tractability. In applications to insurance, it means that a policy can be priced at inception, but its mathematical provision does not admit any analytical expression. An alternative consists to perform simulations. As the provision is the expected discounted difference between benefits and premiums, we have nevertheless to implement a computationally intensive method of simulations in simulations. This article remedies to this issue by showing that properties at birth of the subordinator may be adapted at later times at the condition to observe the filtration of the $\alpha$-stable process before inversion. This assumption allows us to estimate not only the fair premium but also the provision of a wide variety of contracts.

This article contributes to the literature in several directions. Section 3 establishes useful properties of the inverse stable subordinator conditionally to the filtration after the birth of the process. In Section 4, we show that the subordinated chain is solution of a fractional Kolmogorov equation and that waiting times in each state have a Mittag-Leffler distribution. If the chain has an absorbing state, the entry time in this chain is a fractional Phase-Type random variable. We also find the instantaneous transition probabilities of the fractional chain. Section 5 focuses on insurance pricing and on the valuation of provisions. Results are compared with the non-fractional approach introduced in the second section.

\section{The non-fractional model}

In many forms of insurances of persons, benefits and premiums are typically contingent upon transitions of the policy between certain states specified in the contract. The evolution of the insured through these different states can be assimilated to the sample path of a Markov chain. A detailed presentation of Markov chains in discrete and continuous time is available in Chapters 7 and 8 of Rolski et al. (1999). The reader interested by applications of Markov chain in life insurance can refer to lecture notes of Norberg (2002). The book of Denuit and Robert (2007) provides a complete overview of valuation techniques for life and healthcare insurances, based on Markov and semi-Markov chains. We review in this section the classical Markov chain framework that is the foundation of the fractional model studied in Section 5.

The health status of an insured is represented by a continuous-time Markov chain, $\left(\theta_{t}\right)_{t>0}$ taking its value in a finite states space, $E=\left\{e_{1}, \ldots, e_{l}\right\}$, where $e_{k}=(0 \ldots 0,1,0 \ldots 0)^{\top}$ is the $k^{t h}$ unit basis vector of $\mathbb{R}^{l}$. The states correspond e.g. to disability, illness, or death. The probability space is noted $\Omega$ whereas $\left(\mathcal{F}_{t}\right)_{t \geq 0}$ is the natural filtration of $\theta_{t}$ and $P$ is the measure of probability. The probability of transition from state $i$ to $j$ between times $s$ and $t$ is noted

$$
p_{i, j}(s, t)=P\left(\theta_{t}=e_{j} \mid \theta_{s}=e_{i}\right) .
$$

The matrix of these transition probabilities is denoted by $P(s, t)=\left(p_{i, j}(s, t)\right)_{i, j=1, \ldots, l}$. This matrix admits a representation in terms of instantaneous probabilities $Q=\left(q_{i, j}\right)_{i, j=1, \ldots l}$. The $q_{i, j}$ 's are such that $q_{i, j}>0$ for $i \neq j$ and $q_{i, i}=-\sum_{i \neq j} q_{i, j}$. If we remember that the exponential of a matrix $M$ is defined as $\exp (M)=\sum_{k=0}^{\infty} \frac{M^{k}}{k !}, P(s, t)$ is related to $Q$ by the following relation ${ }^{1}$

$$
P(s, t)=\exp (Q(t-s)),
$$

\footnotetext{
${ }^{1}$ Notice that $Q(t-s)$ is the element wise product of $Q$ and $(t-s)$.
} 
and therefore $\frac{\partial P(s, t)}{\partial t}=Q P(s, t)$, which is the Kolmogorov equation. The number of transition from state $i$ to $j \neq i$ is a counting process noted $N_{t}^{i, j}$. The intensity of this process is $\mathbb{E}\left(d N_{t}^{i, j} \mid \mathcal{F}_{t-}\right)=\mathbf{1}_{\left\{\theta_{t-}=e_{i}\right\}} q_{i, j} d t$ where $\mathbf{1}_{\left\{\theta_{t-}=e_{i}\right\}}$ is an indicator variable.

We assume that premiums and annuities are respectively collected or paid in continuous time and depend upon the state of $\theta_{t}$. The vector of premium rates is denoted by $\boldsymbol{c}=\left(c_{1}, \ldots, c_{l}\right)^{\top}$ whereas the annuity rates are $\boldsymbol{a}=\left(a_{1}, \ldots, a_{l}\right)^{\top}$. An insurance contract can also foresee lump sum payments when the insured changes of state. The matrix of these benefits is $D=\left(d_{i, j}\right)_{i, j=1, \ldots, l}$ where $d_{i, j}$ is the amount paid if $\theta_{t}$ switches from state $i$ to $j$. We consider that $d_{j, j}=0$ for $j=1, \ldots, l$. The discount rate is noted $r \in \mathbb{R}^{+}$. If the insurance contract is signed at time 0 and is valid up to $T$, premiums and benefits must fulfill the actuarial equilibrium equation

$$
\begin{aligned}
& \mathbb{E}_{0}\left(\int_{0}^{T} e^{-r u} \theta_{u}^{\top} \boldsymbol{c} d u\right)=\mathbb{E}_{0}\left(\int_{0}^{T} e^{-r u} \theta_{u}^{\top} \boldsymbol{a} d u\right) \\
& \quad+\sum_{i=1}^{l} \sum_{j=1, i \neq j}^{l} \mathbb{E}_{0}\left(\int_{0}^{T} e^{-r u} \mathbf{1}_{\left\{\theta_{u}=e_{i}\right\}} d_{i, j} d N_{u}^{i, j}\right),
\end{aligned}
$$

where $\mathbb{E}_{t}($.$) stands for \mathbb{E}\left(. \mid \mathcal{F}_{t}\right)$. This equation ensures that collected premiums are on average sufficient to fund benefits. At time $t>0$, this equilibrium is not satisfied and the difference between expected discounted benefits and premiums, is the mathematical provision of the contract, noted $V_{t}$,

$$
\begin{aligned}
V_{t}= & \mathbb{E}_{t}\left(\int_{t}^{T} e^{-r(u-t)} \theta_{u}^{\top} \boldsymbol{a} d u\right)+\sum_{i=1}^{l} \sum_{j=1, i \neq j}^{l} \mathbb{E}_{t}\left(\int_{t}^{T} e^{-r(u-t)} \mathbf{1}_{\left\{\theta_{u}=e_{i}\right\}} d_{i, j} d N_{u}^{i, j}\right) \\
& -\mathbb{E}_{t}\left(\int_{t}^{T} e^{-r(u-t)} \theta_{u}^{\top} \boldsymbol{c} d u\right) .
\end{aligned}
$$

This provision is accounted as a liability in the balance sheet of the insurer. Let us denote by $I$ is the identity matrix of dimension $l$. The expectation in Equation (2) can be reformulated as follows:

$$
\begin{aligned}
\mathbb{E}_{t}\left(\int_{t}^{T} e^{-r(u-t)} \theta_{u}^{\top} d u\right) & =\int_{t}^{T} e^{-r(u-t)} \theta_{t}^{\top} P(t, u) d u \\
& =\int_{t}^{T} e^{-r(u-t)} \theta_{t}^{\top} e^{Q(u-t)} d u \\
& =\int_{t}^{T} \theta_{t}^{\top} e^{(Q-r I)(u-t)} d u .
\end{aligned}
$$

The integral of a matrix exponential of the type $e^{M u}$ where $M$ is invertible, is a matrix equal to

$$
\int_{t}^{T} e^{M u} d u=M^{-1}\left(e^{M T}-e^{M t}\right) .
$$

Therefore, we infer that equation (4) is equal to

$$
\mathbb{E}_{t}\left(\int_{t}^{T} e^{-r(u-t)} \theta_{u}^{\top} d u\right)=\theta_{t}^{\top}(Q-r I)^{-1}\left(e^{(Q-r I)(T-t)}-I\right) .
$$

Notice that when $T \rightarrow \infty$, this becomes: $\mathbb{E}_{t}\left(\int_{t}^{\infty} e^{-r(u-t)} \theta_{u}^{\top} d u\right)=\theta_{t}^{\top}(r I-Q)^{-1}$. If $\theta_{0}=e_{k}$, the second expectation in Equation (3) becomes

$$
\mathbb{E}_{t}\left(\int_{t}^{T} e^{-r(u-t)} \mathbf{1}_{\left\{\theta_{u}=e_{i}\right\}} d_{i, j} d N_{u}^{i, j}\right)=\int_{t}^{T} e^{-r(u-t)} p_{k, i}(t, u) q_{i, j} d_{i, j} d u .
$$


Since $d_{j, j}=0$, the double sum of these expectations for $i, j \in\{1, \ldots, l\}$ admits a matrix representation

$$
\begin{aligned}
& \sum_{i=1}^{l} \sum_{j=1, i \neq j}^{l} \mathbb{E}_{t}\left(\int_{t}^{T} e^{-r(u-t)} \mathbf{1}_{\left\{\theta_{u}=e_{i}\right\}} d_{i, j} d N_{u}^{i, j}\right) \\
& =\theta_{t}^{\top}\left(\int_{t}^{T} e^{(Q-r I)(u-t)} Q \otimes D d u\right) \boldsymbol{e} \\
& =\theta_{t}^{\top}(Q-r I)^{-1}\left(e^{(Q-r I)(T-t)}-I\right) Q \otimes D \boldsymbol{e} .
\end{aligned}
$$

where $\otimes$ is the element wise product and $e=(1, \ldots, 1)^{\top}$ is a $l$ vector of ones. From Equations (2), (5) and (6), the provision is then equal to

$$
V_{t}=\theta_{t}^{\top}(Q-r I)^{-1}\left(e^{(Q-r I)(T-t)}-I\right)((\boldsymbol{a}-\boldsymbol{c})+Q \otimes D \boldsymbol{e}) .
$$

We deduce that the fair premium rate $c$ guaranteeing the actuarial equilibrium at inception of the policy is

$$
\theta_{0}^{\top}(Q-r I)^{-1}\left(e^{(Q-r I) T}-I\right) \boldsymbol{c}=\theta_{0}^{\top}(Q-r I)^{-1}\left(e^{(Q-r I) T}-I\right)(\boldsymbol{a}+Q \otimes D \boldsymbol{e})
$$

The fair premium rate if $T=\infty$ is such that

$$
\theta_{0}^{\top}(r I-Q)^{-1} \boldsymbol{c}=\theta_{0}^{\top}(r I-Q)^{-1}(\boldsymbol{a}+Q \otimes D \boldsymbol{e}) .
$$

Notice that for given benefits $\boldsymbol{a}$ and $\boldsymbol{D}$, this equation admits multiple solutions for $\boldsymbol{c}$. One possible solution of Equation (7) is

$$
c=a+Q \otimes D e,
$$

but usually premiums are exclusively paid in a one or two predetermined states. In the remainder of this section, we consider a contract that remains in force till the insured's death. The decease of insured is modeled as an absorbing state, i.e. a state that the Markov chain would not leave after reaching it. If this state is the first one, the matrix of instantaneous probabilities has the form

$$
Q=\left(\begin{array}{ll}
0 & 0 \\
\boldsymbol{b} & B
\end{array}\right)
$$

where $\boldsymbol{b}=\left(b_{1}, \ldots, b_{l-1}\right)^{\top}$ is a $l-1$ vector of non negative components whereas $B=\left(b_{i j}\right)_{i, j=1, \ldots, l-1}$ is a $(l-1) \times(l-1)$ matrix with $b_{i j} \geq 0$ for $i \neq j$ and $b_{i i} \leq 0$. $\mathbf{0}$ is here a null vector of dimension $l-1$. The matrix $B$ and the vector $\boldsymbol{b}$ satisfy the following relation

$$
\boldsymbol{b}=-B \boldsymbol{e}_{l-1}
$$

where $\boldsymbol{e}_{l-1}$ is here a $(l-1)$ vector of ones. This ensures that $Q$ is a matrix of instantaneous probabilities. The time of decease is noted $\zeta$ and is a stopping time with respect to the filtration $\mathcal{F}_{t}$. It is distributed as a phase-type random variable and the probability that the insured survives up to time $t$ is given by

$$
P(\zeta>t)=\theta_{0}^{\top} \exp (t B) \boldsymbol{e}_{l-1}
$$

where $\theta_{0}^{\prime} \in \theta_{0}$ is the vector containing the last $l-1$ values of $\theta_{0}$. The probability density function (pdf) of $\zeta$ is then

$$
f(t)=\theta_{0}^{\prime}{ }^{\top} \exp (t B) \boldsymbol{b} \quad t \geq 0 .
$$

In this particular case, the matrix of benefits paid at transition is given by

$$
D=\left(\begin{array}{cc}
0 & \mathbf{0} \\
\boldsymbol{d} & \bar{D}
\end{array}\right)
$$


where $\boldsymbol{d}$ is a $l-1$ vector and $\bar{D}$ is the $l-1 \times l-1$ identity matrix. One possible solution of Equation (7) is in this case,

$$
\boldsymbol{c}=\boldsymbol{a}+\left(\begin{array}{cc}
0 & \mathbf{0} \\
\boldsymbol{b} \otimes \boldsymbol{d} & B \otimes \bar{D}
\end{array}\right) \boldsymbol{e} .
$$

The Markovian framework presented in this paragraph presents a major drawback: the sojourn time in certain state $j \in\{1, \ldots, l\}$ is distributed as an exponential random variable of parameter $-q_{j, j}$. As the exponential distribution is memoryless, the remaining duration before leaving a state is independent from the time already spent in this state. There exist many solutions to circumvent this drawback. E.g. we could introduce time dependent transition probabilities. In this article, we explore an alternative approach based on stochastic clocks.

\section{Stochastic clocks}

A subordinator is a stochastic process noted $\left(U_{t}\right)_{t>0}$, with positive, non-decreasing sample paths and taking values in $\mathbb{R}^{+}$. We consider here $\alpha$-stable Lévy subordinators. They are Lévy processes with independent and homogeneously distributed increments. We refer the reader to Applebaum (2009) for an introduction to this category of processes. Subordinators can be used directly as stochastic clocks but since their increments are independent, it limits the type of memory (conditionally to $U_{t}$, the sojourn time in a state is still exponential). For this reason, we instead consider a stochastic clock based on an inverse $\alpha$-stable subordinator. In order to understand how we build this inverse, we first review the main features of Lévy subordinators. The process $U_{t}$ is a $\frac{1}{\alpha}$ self-similar process, meaning that for all $a>0$

$$
U_{a t} \stackrel{d}{=}(a t)^{\frac{1}{\alpha}} U_{1}
$$

From the Lévy-Khintchine formula, the Laplace transform of $\alpha$-stable Lévy subordinators has the following structure

$$
\mathbb{E}\left(e^{-\omega U_{t}}\right)=e^{-t \omega^{\alpha}}
$$

The inverse of a Lévy subordinator is a process denoted by $\left(S_{t}\right)_{t \geq 0}$ defined by the following relation

$$
S_{t}=\inf \left\{\tau>0: U_{\tau} \geq t\right\}
$$

It corresponds to the hitting time of $U_{t}$ to the barrier $t$. This inverse is not anymore a Lévy process. However $\left(S_{t}\right)_{t>0}$ is positive and non-decreasing and is then also a subordinator. In the next sections, we use $S_{t}$ as time-change for the Markov chain $\theta_{t}$, i.e. we consider $\theta_{S_{t}}$ instead of $\theta_{t}$.

By construction, the process $S_{t}$ may be constant over relatively short periods of time. Therefore, any process subordinated by $S_{t}$ exhibits motionless periods. In particular for $\theta_{S_{t}}$, this extends on average the time spent in each state and the duration of sojourn has a sub-exponential distribution. We will see in Section 4 that the time-changed Markov chain may be characterized by a fractional differential equation similar to Equation (1).

The remainder of this section reviews the properties of the stochastic clock, needed to evaluate insurance liabilities when the health status of the insured is driven by $\theta_{S_{t}}$ instead of $\theta_{t}$. There is an overlap between results presented in this section and the existing literature on fractional processes. Nevertheless, the literature mainly focuses on properties of $S_{t}$ conditionally to the initial information, at time 0 . These are not adapted for actuarial or financial applications because we must also be capable to evaluate again a contract after its inception e.g. for risk management. Therefore, we extend in this section existing properties by conditioning them to the filtration at later times. Since increments of $S_{t}$ are not independent, their construction requires a particular care and additional information about the process $\left(U_{t}\right)_{t \geq 0}$ is needed.

The natural filtration of $\left(S_{t}\right)_{t \geq 0}$ is denoted by $\left(\mathcal{G}_{t}\right)_{t \geq 0}$. The probability density functions of $U_{t}$ and 
$S_{t}$ are denoted by $p_{U}(t, u)=\frac{d}{d u} P\left(u \leq U_{t} \leq u+d u\right)$ and $g(t, \tau)=\frac{d}{d \tau} P\left(\tau \leq S_{t} \leq \tau+d \tau\right)$. On the other-hand, the self-similarity leads to the relation

$$
\begin{aligned}
P\left(U_{\tau} \leq t\right) & =P\left(\tau^{\frac{1}{\alpha}} U_{1} \leq t\right) \\
& =P\left(U_{1} \leq t \tau^{-\frac{1}{\alpha}}\right) .
\end{aligned}
$$

If we derive this last expression with respect to $t$, we obtain the following scaling property

$$
p_{U}(\tau, t)=\tau^{-\frac{1}{\alpha}} p_{U}\left(1, t \tau^{-\frac{1}{\alpha}}\right) .
$$

This distribution is required for pricing insurance contracts at their inception. However for the calculation of provisions at a later time $s>0$, we have to establish the features of $S_{t}-S_{s}$ conditionally to the available information up to $s$. To the best of our knowledge, most of articles focus on properties of $S_{t}$ seen from time 0 , when $U_{0}=S_{0}=0$. We have then to extend these features when the process $S_{t}$ is considered from a later time. However $S_{t}$ is by nature not Markov and its filtration up to time $s<t$ is not sufficient to draw any conclusions about $S_{t}-S_{s}$. We have then to adjunct additional information about the $\alpha$-stable process that is inverted. In following developments, we show that most of properties available at time 0 admit an extension at later time $s$ if the available information is the value of $S_{s}$ and $U_{s_{s}}$. In this case, we will see in Section 5 that the provision of an insurance contract admits an analytical expression. If $U_{S_{s}}$ is not observed, an average provision can be calculated either by simulating $U_{t}$ and $S_{t}$. Nevertheless, in comparison to an evaluation fully based on a Monte-Carlo method, we do not have to perform simulations in simulations, which are computationally intensive.

Let us recall that $P\left(S_{t} \leq \tau\right)=P\left(U_{\tau} \geq t\right)$. Therefore, for $0 \leq s \leq u \leq t$ and $v \leq \tau$, the cdf of $S_{t}-S_{s}$ is related to the pdf of $U_{t}$ throught the relation

$$
\begin{aligned}
& P\left(S_{t}-S_{s} \leq \tau \mid S_{s}=v, U_{S_{s}}=u\right) \\
& \quad=P\left(S_{t} \leq \tau+v \mid S_{s}=v, U_{S_{s}}=u\right) \\
& \quad=P\left(U_{\tau+v} \geq t \mid S_{s}=v, U_{S_{s}}=u\right) \\
& \quad=P\left(U_{\tau} \geq t-u\right) .
\end{aligned}
$$

If $u>t$ then the clock is stopped between $t$ and $s$ and $P\left(S_{t}-S_{s} \leq \tau \mid S_{s}=v, U_{S_{s}}=u\right)=1$ for all $\tau \geq 0$. As $P\left(U_{\tau} \geq t-u\right)$ does not depend on $v$, this leaves us wondering about the utility of the condition $S_{s}=v$. To understand this, we rewrite the conditional probability (17) as

$$
P\left(S_{t}-v \leq \tau \mid U_{v}=u\right)=P\left(U_{\tau} \geq t-u\right) .
$$

This last equation emphasizes that $P\left(U_{\tau} \geq t-u\right)$ is the cdf of $S_{t}-v$, conditionally to $U_{v}=u$ for all $v \in \mathbb{R}^{+}$. This also explains why $v$ does not explicitely appear in many results detailed in the remainder of this section.

The conditional pdf of $S_{t}-S_{s}$, denoted by $g(t, \tau \mid s, v, u)$ for $t \geq s$, is the derivative of Equation (17) with respect to $\tau$,

$$
\begin{aligned}
g(t, \tau \mid s, v, u) & =\frac{\partial}{\partial \tau} P\left(S_{t}-S_{s} \leq \tau \mid S_{s}=v, U_{S_{s}}=u\right) \\
& =-\frac{\partial}{\partial \tau} P\left(U_{\tau} \leq t-u\right) \\
& =-\frac{\partial}{\partial \tau} \int_{0}^{t-u} p_{U}(\tau, x) d x .
\end{aligned}
$$

The Laplace's transform of $g(. \mid$.$) with respect to time t$ is therefore equal to

$$
\widetilde{g}(\omega, \tau \mid s, v, u)=-\frac{\partial}{\partial \tau} \mathcal{L}\left(\int_{0}^{t-u} p_{U}(\tau, x) d x\right)(\omega),
$$


where $\mathcal{L}\left(\int_{0}^{t-u} p_{U}(\tau, x) d x\right)(\omega)$ is here the Laplace's transform of the integral of $p_{U}($.$) . The process$ $\left(U_{t}\right)_{t \geq 0}$ being strictly positive, its density is null on $\mathbb{R}^{-}$. Integrating by parts leads to the following expression for this Laplace transform,

$$
\begin{aligned}
\mathcal{L} & \left(\int_{0}^{t-u} p_{U}(\tau, x) d x\right)(\omega)=\int_{u}^{\infty} e^{-\omega t} \int_{0}^{t-u} p_{U}(\tau, x) d x d t \\
& =\left[-\frac{e^{-\omega t}}{\omega} \int_{0}^{t-u} p_{U}(\tau, x) d x\right]_{t=u}^{t=\infty}+\frac{1}{\omega} \int_{u}^{\infty} e^{-\omega t} p_{U}(\tau, t-u) d t \\
& =\frac{1}{\omega} e^{-\omega u} \int_{0}^{\infty} e^{-\omega z} p_{U}(\tau, z) d z \\
& =\frac{1}{\omega} e^{-\omega u} \widetilde{p_{U}}(\tau, \omega) .
\end{aligned}
$$

where $\widetilde{p_{U}}(\tau, \omega)=e^{-\tau \omega^{\alpha}}$. From Equation (19), we infer that the Laplace transform of $g($.$) is equal$ to

$$
\widetilde{g}(\omega, \tau \mid s, v, u)=\omega^{\alpha-1} e^{-\omega u-\tau \omega^{\alpha}} .
$$

On the other-hand, from the self-similarity of $U_{t}$ we know that $P\left(U_{\tau} \leq t-u\right)=P\left(U_{1} \leq(t-u) \tau^{-\frac{1}{\alpha}}\right)$. If we derive this last expression with respect to $\tau$ and using Equation (16), we obtain that

$$
\frac{\partial}{\partial \tau} P\left(U_{1} \geq(t-u)(\tau)^{-\frac{1}{\alpha}}\right)=\frac{(t-u)}{\alpha \tau} p_{U}(\tau, t-u),
$$

because $U_{t}$ is $\alpha$ stable. From Equations (18) and (20), we infer an important relation linking the conditional pdf of increments to the pdf of $U_{t}$ :

$$
g(t, \tau \mid s, v, u)=\frac{(t-u)}{\alpha \tau} p_{U}(\tau, t-u) .
$$

In the next proposition, we establish a kind of conditional self-similarity for the density $g(. \mid$.$) :$

Proposition 3.1. For $0 \leq s \leq u \leq t$, the pdf of $S_{t}-S_{s} \mid S_{s}=v, U_{S_{s}}=u$, is self-similar in the following sense:

$$
g(t, \tau \mid s, v, u)=\frac{1}{(t-u)^{\alpha}} g\left(u+1, \frac{\tau}{(t-u)^{\alpha}} \mid s, v, u\right) .
$$

Proof Applying Equation (21) to $g\left(1, \frac{\tau}{t^{\alpha}}\right)$ leads to the relation

$$
g\left(u+1, \frac{\tau}{(t-u)^{\alpha}} \mid s, v, u\right)=\frac{(t-u)^{\alpha}}{\alpha \tau} p_{U}\left(\frac{\tau}{(t-u)^{\alpha}}, 1\right) .
$$

Using twice the self similarity of $p_{U}(\tau, t)=\tau^{-\frac{1}{\alpha}} p_{U}\left(1, t \tau^{-\frac{1}{\alpha}}\right)$ allows us to infer that

$$
\begin{aligned}
p_{U}\left(\frac{\tau}{(t-u)^{\alpha}}, 1\right) & =(t-u) \tau^{-\frac{1}{\alpha}} p_{U}\left(1,(t-u) \tau^{-\frac{1}{\alpha}}\right) \\
& =(t-u) p_{U}(\tau,(t-u)) .
\end{aligned}
$$

Combining Equations (23) and (24) provides the equality

$$
\begin{aligned}
g\left(u+1, \frac{\tau}{(t-u)^{\alpha}} \mid s, v, u\right) & =(t-u)^{\alpha} \frac{(t-u)}{\alpha \tau} p_{U}(\tau,(t-u)) \\
& =(t-u)^{\alpha} g(t, \tau \mid s, v, u),
\end{aligned}
$$

which allows us to conclude.

Notice that for $0 \leq s \leq t \leq u$, the Markov chain is motionless since the stochastic clock is stopped. Using this last result allows us to calculate the moments of $S_{u+1}-S_{s}$. 
Proposition 3.2. for $0 \leq s \leq u \leq t$, the moments of $S_{u+1}-S_{s}$, conditionally to $S_{s}$ and $U_{S_{s}}$, for $n \in \mathbb{N}$ are given by

$$
\mathbb{E}\left(\left(S_{u+1}-S_{s}\right)^{n} \mid S_{s}=v, U_{S_{s}}=u\right)=\frac{\Gamma(n+1)}{\Gamma(n \alpha+1)} .
$$

Proof Using the self-similarity property of Proposition 3.1, the Laplace transform of $U_{t-u}$ is rewritten as

$$
\begin{aligned}
e^{-(t-u) \omega^{\alpha}} & =\int_{0}^{\infty} e^{-\omega x} p_{U}(t-u, x) d x \\
& =\int_{0}^{\infty} e^{-\omega x}(t-u)^{-\frac{1}{\alpha}} p_{U}\left(1, x(t-u)^{-\frac{1}{\alpha}}\right) d x .
\end{aligned}
$$

From Equations (21) and (16), we infer that

$$
\begin{aligned}
p_{U}\left(1, x(t-u)^{-\frac{1}{\alpha}}\right) & =(t-u)^{\frac{1}{\alpha}} p_{U}(t-u, x) \\
& =\frac{\alpha}{x}(t-u)^{\frac{1}{\alpha}+1} g(u+x,(t-u) \mid s, v, u) .
\end{aligned}
$$

The Laplace transform of $U_{t-u}$ becomes then

$$
e^{-(t-u) \omega^{\alpha}}=\alpha \int_{0}^{\infty} e^{-\omega x} \frac{(t-u)}{x} g(u+x, t-u \mid s, v, u) d x .
$$

From Equation (22) of Proposition 3.1, we rewrite this last integral as

$$
e^{-(t-u) \omega^{\alpha}}=\alpha \int_{0}^{\infty} e^{-\omega x} \frac{(t-u)}{x^{\alpha+1}} g\left(u+1,(t-u) x^{-\alpha} \mid s, v, u\right) d x .
$$

Multiplying the left hand side by $(t-u)^{n-1}$ and integrating it with respect to $t$ give us (as $\Gamma(z)=$ $\left.\int t^{z-1} e^{-t} d t\right)$

$$
\begin{aligned}
\int_{u}^{\infty}(t-u)^{n-1} e^{-(t-u) \omega^{\alpha}} d t & =\int_{0}^{\infty} t^{n-1} e^{-t \omega^{\alpha}} d t \\
& =\frac{1}{\omega^{n \alpha}} \int_{0}^{\infty}\left(\omega^{\alpha} t\right)^{n-1} e^{-t \omega^{\alpha}} d\left(\omega^{\alpha} t\right) \\
& =\frac{\Gamma(n)}{\omega^{n \alpha}}
\end{aligned}
$$

We multiply by $(t-u)^{n-1}$ the right hand side of Equation (26) and integrate it, using again the self-similarity Equation (22):

$$
\begin{aligned}
& \alpha \int_{u}^{\infty} \int_{0}^{\infty} e^{-\omega x} \frac{(t-u)^{n}}{x^{\alpha+1}} g\left(u+1,(t-u) x^{-\alpha} \mid s, v, u\right) d x d t \\
& \quad=\alpha \int_{0}^{\infty} \int_{0}^{\infty} e^{-\omega x} \frac{\tau^{n}}{x^{\alpha+1}} g\left(u+1, \tau x^{-\alpha} \mid s, v, u\right) d x d \tau \\
& \quad=\alpha \int_{0}^{\infty} \int_{0}^{\infty} e^{-\omega x} \frac{x^{n \alpha}}{x^{\alpha+1}}\left(\tau x^{-\alpha}\right)^{n} g\left(u+1, \tau x^{-\alpha} \mid s, v, u\right) d x d \tau \\
& =\alpha \int_{0}^{\infty} e^{-\omega x} \frac{x^{n \alpha-1}}{x^{\alpha}} \int_{0}^{\infty}\left(\tau x^{-\alpha}\right)^{n} g\left(u+1, \tau x^{-\alpha} \mid s, v, u\right) d \tau d x \\
& =\alpha \int_{0}^{\infty} e^{-\omega x} x^{n \alpha-1} \int_{0}^{\infty} z^{n} g(u+1, z \mid s, v, u) d z d x \\
& =\alpha \int_{0}^{\infty} e^{-\omega x} x^{n \alpha-1} d x \mathbb{E}\left(\left(S_{u+1}-S_{v}\right)^{n} \mid S_{s}=v, U_{S_{s}}=u\right) \\
& =\alpha \frac{\Gamma(n \alpha)}{\omega^{n \alpha}} \mathbb{E}\left(\left(S_{u+1}-S_{v}\right)^{n} \mid S_{s}=v, U_{S_{s}}=u\right) .
\end{aligned}
$$


Combining this result with Equations (26), we obtain that

$$
\mathbb{E}\left(\left(S_{u+1}-S_{s}\right)^{n} \mid S_{s}=v, U_{S_{s}}=u\right)=\frac{\Gamma(n)}{\Gamma(n \alpha) \alpha}=\frac{\Gamma(n+1)}{\Gamma(n \alpha+1)},
$$

which is Equation (25).

From moments of $S_{u+1}-S_{s}$, we infer the Laplace transform of the stochastic clock increments.

Corollary 3.3. for $0 \leq s \leq u \leq t$, the Laplace's transform of $S_{t}-S_{s}$ conditionally to $S_{s}$ and $U_{S_{s}}$ is:

$$
\mathbb{E}\left(e^{-\omega\left(S_{t}-S_{s}\right)} \mid S_{s}=v, U_{S_{s}}=u\right)=E_{\alpha}\left(-\omega(t-u)^{\alpha}\right),
$$

where $E_{\alpha}(X)=\sum_{n=0}^{\infty} \frac{X^{n}}{\Gamma(n \alpha+1)}$ is the Mittag-Leffler function. The moments of order $n \in \mathbb{N}$ are given by

$$
\mathbb{E}\left(\left(S_{t}-S_{s}\right)^{n} \mid S_{s}=v, U_{S_{s}}=u\right)=\frac{\Gamma(n+1)}{\Gamma(n \alpha+1)}(t-u)^{n \alpha} .
$$

If $A$ is a $l \times l$ matrix such that $\lim _{t \rightarrow \infty} A^{t}$ exists, then

$$
\mathbb{E}\left(e^{A\left(S_{t}-S_{s}\right)} \mid S_{s}=v, U_{S_{s}}=u\right)=\boldsymbol{E}_{\alpha}\left(A(t-u)^{\alpha}\right),
$$

where $\boldsymbol{E}_{\alpha}(X)=\sum_{n=0}^{\infty} \frac{X^{n}}{\Gamma(n \alpha+1)}$ is the matrix Mittag-Leffler function.

Proof Using again the self-similarity property 3.1 and performing a change of variable, we rewrite the Laplace transform as

$$
\begin{aligned}
& \mathbb{E}\left(e^{-\omega\left(S_{t}-S_{s}\right)} \mid S_{s}=v, U_{S_{s}}=u\right)=\int_{0}^{\infty} e^{-\omega \tau} g(t, \tau \mid s, v, u) d \tau \\
& \quad=\int_{0}^{\infty} e^{-\omega \tau} \frac{1}{(t-u)^{\alpha}} g\left(u+1, \frac{\tau}{(t-u)^{\alpha}} \mid s, v, u\right) d \tau \\
& \quad=\int_{0}^{\infty} e^{-\omega \gamma(t-u)^{\alpha}} g(u+1, \gamma \mid s, v, u) d \gamma \\
& =\mathbb{E}\left(e^{-\omega(t-u)^{\alpha}\left(S_{u+1}-S_{s}\right)} \mid S_{s}=v, U_{S_{s}}=u\right) .
\end{aligned}
$$

The Taylor's development of the Laplace's transform around $\omega=0$ combined with Proposition 3.2 allow us to rewrite this transform as the following infinite sum

$$
\begin{aligned}
\mathbb{E}\left(e^{-\omega(t-u)^{\alpha}\left(S_{u+1}-S_{s}\right)} \mid S_{s}=v, U_{S_{s}}=u\right) \\
\quad=\sum_{n=0}^{\infty} \frac{\mathbb{E}\left(\left(S_{u+1}-S_{s}\right)^{n} \mid S_{s}=v, U_{S_{s}}=u\right)}{\Gamma(n+1)}\left(-\omega(t-u)^{\alpha}\right)^{n} \\
\quad=\sum_{n=0}^{\infty} \frac{\left(-\omega(t-u)^{\alpha}\right)^{n}}{\Gamma(n \alpha+1)}
\end{aligned}
$$

which is precisely the Mittag-Leffler function at point $-\omega(t-u)^{\alpha}$. Moments are obtained by deriving the Laplace transform. Equation (30) is proved in the same way as the first statement.

Notice that for $0 \leq s \leq t \leq u, \mathbb{E}\left(e^{-\omega\left(S_{t}-S_{s}\right)} \mid S_{s}=v, U_{S_{s}}=u\right)=1$. To conclude this section, we calculate the conditional expectation of the infinitesimal variation of $S_{t}$.

Proposition 3.4. For $0 \leq s \leq u \leq t, v \geq 0$, the expectation of $\frac{d S_{t}}{d t}$ is equal to

$$
\mathbb{E}\left(\frac{d S_{t}}{d t} \mid S_{s}=v, U_{S_{s}}=u\right)=\frac{\alpha(t-u)^{\alpha-1}}{\Gamma(\alpha+1)} .
$$



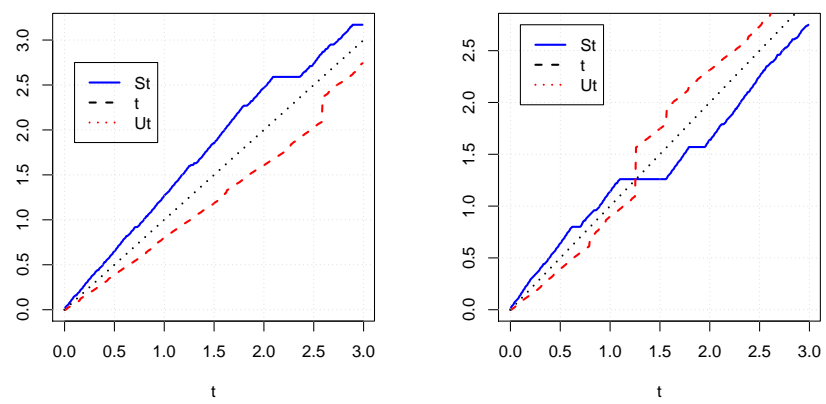

Figure 1: Two sample paths of an inverse $\alpha$ stable process $(\alpha=0.95)$.

Proof From corollary 3.3, we deduce that

$$
\begin{aligned}
\mathbb{E} & \left(S_{t+\Delta}-S_{t} \mid S_{s}=v, U_{S_{s}}=u\right) \\
& =\mathbb{E}\left(S_{t+\Delta}-S_{s}+S_{s}-S_{t} \mid S_{s}=v, U_{S_{s}}=u\right) \\
& =\frac{(t+\Delta-u)^{\alpha}-(t-u)^{\alpha}}{\Gamma(\alpha+1)} .
\end{aligned}
$$

Taking the limit leads to Equation (31).

Figure 1 presents two simulated sample paths of an $\alpha$ stable process and of its inverse when $\alpha=0.95$. As expected, we observe periods over which $S_{t}$ is constant. These periods are due to sharp increases of $U_{t}$.

\section{The fractional chain}

In a similar manner to Section 2, we consider in the sequel that the insured's health status is represented by a Markov chain observed on the random time scale defined by $S_{t}$. The time-changed chain, noted $\left(\theta_{S_{t}}\right)_{t>0}$ is no more a Markov process but presents several interesting properties developed in this section. The natural filtration of the inverse Lévy subordinator $S_{t}$ is denoted by $\left(\mathcal{G}_{t}\right)_{t>0}$ and is independent from the filtration $\left(\mathcal{F}_{t}\right)_{t>0}$ of $\left(\theta_{t}\right)_{t>0}$. The subordinated Markov chain $\theta_{S_{t}}$ is then adapted to a filtration $\mathcal{G}_{t} \vee \mathcal{F}_{S_{t}}$. This is the smallest filtration at the intersection of $\mathcal{G}_{t}$ and $\mathcal{F}_{S_{t}}$. By definition, the filtration of $S_{t}$ is embedded into the filtration of $U_{t}$ up to time $S_{t}$.

For $0 \leq s \leq u \leq t$ and $v \leq \tau$, we denote by $P(t \mid s, v, u)$ the matrix of transition probabilities of a time-changed Markov chain,

$$
P(t \mid s, v, u)=\left(P\left(\theta_{S_{t}}=e_{j} \mid S_{s}=v, U_{s}=u, \theta_{S_{s}}=e_{i}\right)\right)_{i, j=1, \ldots, l} .
$$

From Equation (1), this matrix is equal to the following conditional expectation

$$
\begin{aligned}
P(t \mid s, v, u) & =\mathbb{E}\left(P\left(S_{s}, S_{t}\right) \mid S_{s}=v, U_{S_{s}}=u\right) \\
& =\mathbb{E}\left(e^{Q\left(S_{t}-S_{s}\right)} \mid S_{s}=v, U_{S_{s}}=u\right) .
\end{aligned}
$$

From Corollary 3.3, we immediately infer that $P(t \mid s, v, u)$ is a matrix Mittag-Leffler function

$$
P(t \mid s, v, u)=\left\{\begin{array}{ll}
\boldsymbol{E}_{\alpha}\left(Q(t-u)^{\alpha}\right) & 0 \leq s \leq u \leq t \\
I & 0 \leq s \leq t \leq u
\end{array} .\right.
$$

The second statement in this equation emphasizes that the chain is motionless when $s \leq t \leq u$. This allows us to underline the link with fractional calculus. The Caputo's derivative of order 
$\alpha \in] 0,1\left[\right.$ for a function $h(x): \mathbb{R}^{+} \rightarrow \mathbb{R}, \mathcal{C}^{1}$ with respect to $t$ is defined by

$$
\frac{\partial^{\alpha} h(x)}{\partial x^{\alpha}}=\frac{1}{\Gamma(1-\alpha)}\left(\frac{\partial}{\partial x} \int_{0}^{x}(x-s)^{-\alpha} h(s) d s-\frac{h(0)}{x^{\alpha}}\right) .
$$

An alternative writing is

$$
\frac{\partial^{\alpha} h(x)}{\partial x^{\alpha}}=\frac{1}{\Gamma(1-\alpha)} \int_{0}^{x}(x-s)^{-\alpha} \frac{\partial h(s)}{\partial s} d s .
$$

When $\alpha=1$, this derivative corresponds to the derivative with respect to time. Let $\tilde{h}(\omega)$ be the usual Laplace's transform of a function $h(x)$ with respect to $x$. A direct calculation shows that $\widetilde{\frac{\partial^{\alpha} h}{\partial x^{\alpha}}}(\omega)$, the Laplace's transform of the Caputo's derivative $\frac{\partial^{\alpha}}{\partial x^{\alpha}} h(x)$, is equal to

$$
\frac{\widetilde{\partial^{\alpha} h}}{\partial x^{\alpha}}(\omega)=\omega^{\alpha} \tilde{h}(\omega)-\omega^{\alpha-1} h(0)
$$

which reduces to the familiar form when $\alpha=1$. The next proposition shows that the transition matrix is solution of a fractional Kolmogorov equation.

Proposition 4.1. Let us momentarily denote by $P(x)=P(u+x \mid s, v, u)$ the conditional matrix of transition probabilities from $\theta_{S_{s}}$ to $\theta_{S_{u+x}}$ with $x \geq 0$. This matrix is solution of the fractional differential equation

$$
\frac{\partial^{\alpha} P(x)}{\partial x^{\alpha}}=Q P(x)
$$

where the derivative is the Caputo's derivative (35).

Proof From Equation (35) and by definition of the matrix Mittag-Leffler function,we deduce that the fractional derivative of $P(x)$ is

$$
\frac{\partial^{\alpha} P(x)}{\partial x^{\alpha}}=\frac{1}{\Gamma(1-\alpha)} \sum_{n=1}^{\infty} \frac{n \alpha Q^{n}}{\Gamma(n \alpha+1)} \int_{0}^{x}(x-s)^{-\alpha} s^{\alpha n-1} d s .
$$

If we perform the change of variable $s=x z$, the integral in the right hand term becomes

$$
\begin{aligned}
\int_{0}^{x}(x-s)^{-\alpha} s^{\alpha n-1} d s & =x^{(n-1) \alpha} \int_{0}^{1}(1-z)^{-\alpha} z^{n \alpha-1} d z \\
& =x^{(n-1) \alpha} B(n \alpha, 1-\alpha)
\end{aligned}
$$

where $B(x, y)=\int_{0}^{1} z^{x-1}(1-z)^{y-1} d z$ is the Beta function. Since the Beta function is a ratio of Gamma functions,

$$
B(n \alpha, 1-\alpha)=\frac{\Gamma(n \alpha) \Gamma(1-\alpha)}{\Gamma(n \alpha+1-\alpha)},
$$

we deduce that $P(x)$ satisfies Equation (36).

Fractional derivatives are essentially analytic continuation of the concept of the differential operator (or the antiderivative) into a unified differintegral operator, in the same way that the gamma function is the analytic continuation of the factorial function, so grasping for intuitive explanations isn't easy. It is used in physics of materials and replaces the gradient e.g. for porous materials. They present by nature strong irregularities and when we want to explain the evolution of heat in such a material, the derivative with respect to time $(\alpha=1)$ in the heat equation, is replaced by a fractional one with $\alpha<1$. Brociek et al. (2017) illustrate this for porous aluminum. Compared to non-porous aluminum, its conductivity is lower and the heat diffuses less quickly than in the porous metal. In view of Equation (36), when $\alpha=1$, the chain is a Markov process and transition times are exponential random variables. When $\alpha<1$, as for the non-porous aluminum, transitions 
are slowed down. This point will be confirmed in Corollary 4.2 that requires some intermediate developments.

If the Markov chain has a first state that is absorbing, its matrix of transition probabilities has a structure by blocks such as in Equation (10). In this case, we denote by $\zeta$ the remaining time before $\theta_{S_{t}}$ enters into this absorbing state. The probability that the process $\theta_{S_{t}}$ has not reached this state at time $t$ is noted

$$
P\left(\zeta>t-s \mid v, u, \theta_{v}\right)=P\left(\theta_{S_{t}} \neq e_{1} \mid S_{s}=v, U_{S_{s}}=u, \theta_{S_{s}}=\theta_{v}\right) .
$$

Let us denote $\theta_{v}^{\prime} \subset \theta_{v}$ the subvector of the last $l-1$ elements of $\theta_{v}$ and $\boldsymbol{e}_{l-1}$ a $l-1$ vector of ones. Given that

$$
Q^{n}=\left(\begin{array}{cc}
0 & \mathbf{0} \\
B^{n-1} \boldsymbol{b} & B^{n}
\end{array}\right)
$$

and from Equation (33), the probability $P\left(\zeta>t-s \mid v, u, \theta_{v}\right)$ is given by

$$
\begin{aligned}
P\left(\zeta>t-s \mid v, u, \theta_{v}\right) & =\theta_{v}^{\top} \boldsymbol{E}_{\alpha}\left(Q(t-u)^{\alpha}\right)\left(\begin{array}{c}
0 \\
\boldsymbol{e}_{l-1}
\end{array}\right) \\
& =\theta_{v}^{\top}\left(\sum_{n=0}^{\infty} \frac{B^{n}(t-u)^{n \alpha}}{\Gamma(n \alpha+1)}\right) \boldsymbol{e}_{l-1} \\
& =\theta_{v}^{\prime} \boldsymbol{E}_{\alpha}\left(B(t-u)^{\alpha}\right) \boldsymbol{e}_{l-1} .
\end{aligned}
$$

Corollary 4.2. At time $s$, the remaining sojourn time of $\theta_{S_{t}}$ in the $j^{\text {th }}$ state, denoted by $\zeta_{j}$, is distributed according to a Mittag-Leffler random variable i.e. its cumulative distribution function is given by

$$
P\left(\zeta_{j} \leq t-s \mid S_{s}=v, U_{S_{s}}=u, \theta_{S_{s}}=e_{j}\right)=1-E_{\alpha}\left(-\lambda_{j}(t-u)^{\alpha}\right),
$$

where $\lambda_{j}=\sum_{i \neq j} q_{i j}$ and for $0 \leq s \leq u \leq t$.

Proof Let us define a Markov chain, $\left(\gamma_{t}\right)_{t \geq 0}$ with two states. The second state corresponds to the sojourn of $\theta_{t}$ in the $j^{t h}$ state and the first state to all other events. The first state is absorbing by definition. The transition matrix of $\gamma$ is therefore

$$
Q^{\gamma}=\left(\begin{array}{cc}
0 & 0 \\
\lambda_{i} & -\lambda_{i}
\end{array}\right)
$$

From equation (37), we infer that

$$
P\left(\zeta_{j} \geq t-s \mid S_{s}=v, U_{S_{s}}=u, \theta_{S_{s}}=e_{j}\right)=E_{\alpha}\left(-\lambda_{j}(t-u)^{\alpha}\right),
$$

which is the desired result.

Notice that Albrecher et al. (2020) establish the pdf of the fractional Phase-Type process by considering directly a chain with waiting times that are Mittag-Leffler distributed. In this article, we find this pdf with an alternative approach that is subordination. The next corollary is needed in later developments and relates the derivative of the matrix Mittag-Leffler function to the conditional density of $S_{t}$.

Corollary 4.3. For $0 \leq s \leq u, v>0$ and $Q$ a transition matrix, we have that

$$
\begin{aligned}
& Q \int_{0}^{\infty} z g\left(u+x, z \mid S_{s}=v, U_{S_{s}}=u\right) e^{Q z} d z \\
& =\frac{x}{\alpha} \frac{d \boldsymbol{E}_{\alpha}\left(Q x^{\alpha}\right)}{d x} .
\end{aligned}
$$


Proof We momentarily denote the conditional density $g\left(., . \mid S_{s}=v, U_{S_{s}}=u\right)$ by $g_{s, u, v}(.,$.$) .$ We expand the matrix exponential and use Corollary 3.3, in order to rewrite the integral

$$
\begin{aligned}
Q \int_{0}^{\infty} z g_{s, u, v}(u+x, z) e^{Q z} d z=\sum_{n=0}^{\infty} \frac{Q^{n+1}}{n !} \int_{0}^{\infty} z^{n+1} g_{s, u, v}(u+x, z) d z \\
\quad=\sum_{n=0}^{\infty} \frac{Q^{n+1}}{n !} \mathbb{E}\left(\left(S_{u+x}-S_{s}\right)^{n+1} \mid S_{s}=v, U_{S_{s}}=u\right) \\
\quad=\sum_{n=1}^{\infty} \frac{Q^{n} n}{\Gamma(n \alpha+1)} x^{n \alpha} .
\end{aligned}
$$

On the other hand, by definition of the matrix Mittag-Leffler function, we have

$$
\begin{aligned}
\frac{x}{\alpha} \frac{d}{d x} \boldsymbol{E}_{\alpha}\left(Q x^{\alpha}\right) & =\frac{x}{\alpha} \sum_{n=0}^{\infty} \frac{d}{d x}\left(\frac{Q^{n} x^{n \alpha}}{\Gamma(n \alpha+1)}\right) \\
& =\sum_{n=1}^{\infty} \frac{k Q^{n} x^{n \alpha}}{\Gamma(n \alpha+1)} .
\end{aligned}
$$

Note that this result is also a direct consequence of Equation (30).

In order to ligthen notations in further developments, we denote by $\mathbb{E}_{t, u, v}($.$) , the expectation$ conditionally to the knowledge of $S_{t}=v, U_{S_{t}}=u$ and the state of the fractional chain at time $t$, $\theta_{S_{t}}=\theta_{v}$,

$$
\mathbb{E}_{t, u, v}(.)=\mathbb{E}\left(. \mid S_{t}=v, U_{S_{t}}=u, \theta_{S_{t}}=\theta_{v}\right) .
$$

Let us recall that $P\left(S_{t} \leq \tau\right)=P\left(U_{\tau} \geq t\right)$. Therefore, for $0 \leq s \leq u \leq t_{1} \leq t_{2}$ and $v \leq \tau_{1} \leq \tau_{2}$, the joint conditional cdf of $S_{t_{1}}$ and $S_{t_{2}}$ is equal to

$$
\begin{aligned}
& P\left(S_{t_{1}} \leq \tau_{1}, S_{t_{2}} \leq \tau_{2} \mid S_{s}=v, U_{S_{s}}=u\right) \\
& \quad=P\left(U_{\tau_{1}} \geq t_{1}, U_{\tau_{2}} \geq t_{2} \mid S_{s}=v, U_{S_{s}}=u\right) \\
& \quad=P\left(U_{\tau_{1}-v} \geq t_{1}-u, U_{\tau_{2}-v} \geq t_{2}-u\right) .
\end{aligned}
$$

We denote by $h\left(t_{1}, \tau_{1}, t_{2}, \tau_{2} \mid s, v, u\right)$ the bivariate density of the pair $\left(S_{t_{1}}, S_{t_{2}}\right)$ conditional to $S_{s}=v$ and $U_{S_{s}}=u$. The next proposition allows us later to calculate the matrix of transition probabilities from times $t$ to $t+\Delta$, conditionally up to information available at time $S_{s}$. This is also a matrix version of the proposition 3.1 in Leonenko et al. (2013).

Proposition 4.4. For $0 \leq s \leq u \leq t_{1} \leq t_{2}$, the expectation of $e^{Q\left|S_{t_{2}}-S_{t_{1}}\right|}$ conditionally to $S_{s}=v$ and $U_{S_{s}}=u$ is equal to

$$
\begin{aligned}
\mathbb{E}_{s, u, v}\left(e^{Q\left|S_{t_{2}}-S_{t_{1}}\right|}\right) & =\frac{-\alpha Q}{\Gamma(\alpha+1)} \int_{0}^{t_{1}-u} y^{\alpha-1} \boldsymbol{E}_{\alpha}\left(Q\left(t_{2}-y-u\right)^{\alpha}\right) d y \\
& +\boldsymbol{E}_{\alpha}\left(Q\left(t_{2}-u\right)^{\alpha}\right) .
\end{aligned}
$$

Proof We momentarily denote by $h(\mu, \lambda)=h\left(t_{1}, \mu, t_{2}, \lambda \mid s, v, u\right)$ and $H(\mu, \lambda)$, the bivariate pdf and cdf of the pair $\left(S_{t_{1}}, S_{t_{2}}\right)$ conditionally to $S_{s}=v$ and $U_{S_{s}}=u$. By definition $H(\mu, \infty)=$ $P\left(S_{t_{1}} \leq \mu \mid S_{s}=v, U_{S_{s}}=u\right), H(\infty, \lambda)=P\left(S_{t_{2}} \leq \lambda \mid S_{s}=v, U_{S_{s}}=u\right)$ and $H(\infty, \infty)=1$. The expectation is hence equal to

$$
\mathbb{E}_{s, u, v}\left(e^{Q\left|S_{t_{2}}-S_{t_{1}}\right|}\right)=\int_{0}^{\infty} \int_{0}^{\infty} \underbrace{e^{Q|\lambda-\mu|}}_{f(\mu, \lambda)} H(d \mu, d \lambda) .
$$


Using a bivariate by part integration leads to

$$
\begin{aligned}
\int_{0}^{\infty} \int_{0}^{\infty} f(\mu, \lambda) H(d \mu, d \lambda)= & \int_{0}^{\infty} \int_{0}^{\infty} H([\mu, \infty] \times[\lambda, \infty]) f(d \mu, d \lambda) \\
& +\int_{0}^{\infty} H([\mu, \infty] \times[0, \infty]) f(d \mu, 0) \\
& +\int_{0}^{\infty} H([0, \infty] \times[\lambda, \infty]) f(0, d \lambda) \\
& +f(0,0) H([0, \infty] \times[0, \infty]),
\end{aligned}
$$

where $f(0,0) H([0, \infty] \times[0, \infty])=1$. On the other hand,

$$
\begin{aligned}
H([\mu, \infty] \times[0, \infty]) & =P\left(S_{t_{1}} \geq \mu \mid S_{s}=v, U_{S_{s}}=u\right) \\
& =1-P\left(S_{t_{1}} \leq \mu \mid S_{s}=v, U_{S_{s}}=u\right),
\end{aligned}
$$

therefore, the second term in Equation (40) becomes

$$
\begin{aligned}
& \int_{0}^{\infty} H([\mu, \infty] \times[0, \infty]) f(d \mu, 0) \\
& =Q \int_{0}^{\infty} e^{Q \mu}\left(1-P\left(S_{t_{1}} \leq \mu \mid S_{s}=v, U_{S_{s}}=u\right)\right) d \mu \\
& =\left[e^{Q \mu}\left(1-P\left(S_{t_{1}} \leq \mu \mid S_{s}=v, U_{S_{s}}=u\right)\right)\right]_{0}^{\infty}+\mathbb{E}_{s, u, v}\left(e^{Q S_{t_{1}}}\right) \\
& =\boldsymbol{E}_{\alpha}\left(Q\left(t_{1}-u\right)^{\alpha}\right)-I .
\end{aligned}
$$

In a similar way, we find that the third term in Equation (40) is

$$
\int_{0}^{\infty} H([0, \infty] \times[\lambda, \infty]) f(0, d \lambda)=\boldsymbol{E}_{\alpha}\left(Q\left(t_{2}-u\right)^{\alpha}\right)-I .
$$

The first term of Equation (40) is a double integral. Since $S_{t}$ is increasing and discontinuous and that $P_{s, u, v}\left(\mu \leq S_{t_{1}}, \lambda \leq S_{t_{2}}\right)=P_{s, u, v}\left(\mu \leq S_{t_{1}}\right)$ for $\mu>\lambda$, this integral may be split as follows:

$$
\begin{aligned}
\int_{0}^{\infty} & \int_{0}^{\infty} P_{s, u, v}\left(\mu \leq S_{t_{1}}, \lambda \leq S_{t_{2}}\right) f(d \mu, d \lambda)= \\
& -\int_{0}^{\infty} \int_{0}^{\lambda} P_{s, u, v}\left(\mu \leq S_{t_{1}}, \lambda \leq S_{t_{2}}\right) Q^{2} e^{Q(\lambda-\mu)} d \mu d \lambda \\
& +\int_{0, u=\lambda}^{\infty} P_{s, u, v}\left(\mu \leq S_{t_{1}}, \lambda \leq S_{t_{2}}\right) f(d \mu, d \lambda) \\
& -\int_{0}^{\infty} \int_{\lambda}^{\infty} P_{s, u, v}\left(\mu \leq S_{t_{1}}\right) Q^{2} e^{Q(\mu-\lambda)} d \mu d \lambda .
\end{aligned}
$$

Integrating by parts allows us to rewrite the third term of Equation (41),

$$
\begin{aligned}
-\int_{0}^{\infty} & \int_{\lambda}^{\infty} P_{s, u, v}\left(\mu \leq S_{t_{1}}\right) Q^{2} e^{Q(\mu-\lambda)} d \mu d \lambda \\
= & -\int_{0}^{\infty} P_{s, u, v}\left(\mu \leq S_{t_{1}}\right) \int_{0}^{\mu} Q^{2} e^{Q(\mu-\lambda)} d \lambda d \mu \\
= & -Q \int_{0}^{\infty} P_{s, u, v}\left(\mu \leq S_{t_{1}}\right)\left(I-e^{Q \mu}\right) d \mu \\
= & -Q \int_{0}^{\infty} P_{s, u, v}\left(\mu \leq S_{t_{1}}\right) d \mu-Q \int_{0}^{\infty} P_{s, u, v}\left(\mu \leq S_{t_{1}}\right) e^{Q \mu} d \mu .
\end{aligned}
$$

The first and second integrals of (42) are respectively equal to

$$
\begin{aligned}
-Q \int_{0}^{\infty} P_{s, u, v}\left(\mu \leq S_{t_{1}}\right) d \mu & =-Q \mathbb{E}_{s, u, v}\left(S_{t_{1}}\right) \\
& =-Q \frac{\left(t_{1}-u\right)^{\alpha}}{\Gamma(1+\alpha)}
\end{aligned}
$$


and

$$
Q \int_{0}^{\infty} P_{s, u, v}\left(\mu \leq S_{t_{1}}\right) e^{Q \mu} d \mu=I-\boldsymbol{E}_{\alpha}\left(Q\left(t_{1}-u\right)^{\alpha}\right) .
$$

The third term of Equation (41) is hence given by

$$
\begin{gathered}
-\int_{0}^{\infty} \int_{\lambda}^{\infty} P_{s, u, v}\left(\mu \leq S_{t_{1}}\right) Q^{2} e^{Q(\mu-\lambda)} d \mu d \lambda \\
=-Q \frac{\left(t_{1}-u\right)^{\alpha}}{\Gamma(1+\alpha)}+I-\boldsymbol{E}_{\alpha}\left(Q\left(t_{1}-u\right)^{\alpha}\right) .
\end{gathered}
$$

Since for $\mu=\lambda$, the following function

$$
f(d \mu, \lambda)=Q e^{Q(\mu-\lambda)} \mathbf{1}_{\{\mu>\lambda\}}-Q e^{Q(\lambda-\mu)} \mathbf{1}_{\{\mu<\lambda\}}
$$

has a jump of $-2 Q$, the second term of Equation (41) is given by

$$
\begin{aligned}
\int_{\mu=\nu}^{\infty} P_{s, u, v}\left(\mu \leq S_{t_{1}}, \lambda \leq S_{t_{2}}\right) f(d \mu, d \lambda) & =-2 Q \int_{0}^{\infty} P_{s, u, v}\left(\mu \leq S_{t_{1}}\right) d \mu \\
& =-2 Q \mathbb{E}_{s, u, v}\left(S_{t_{1}}\right) \\
& =-2 Q \frac{\left(t_{1}-u\right)^{\alpha}}{\Gamma(1+\alpha)}
\end{aligned}
$$

On the other hand, for $\mu \leq \lambda$ and $t_{1} \leq t_{2}$, given that $U_{t}$ has independent self-similar increments, the cdf of $\left(S_{t_{1}}, S_{t_{2}}\right)$ is such that

$$
\begin{aligned}
P_{s, u, v}\left(\mu \leq S_{t_{1}}, \lambda \leq S_{t_{2}}\right) & =P\left(U_{\mu-v} \leq t_{1}-u, U_{\lambda-v} \leq t_{2}-u\right) \\
& =P\left(U_{\mu-v} \leq t_{1}-u, U_{\mu-v}+\left(U_{\lambda-v}-U_{\mu-v}\right) \leq t_{2}-u\right) \\
& =\int_{y=0}^{t_{1}-u} p_{U}(\mu-v, y) d y \int_{0}^{t_{2}-y-u} p_{U}(\lambda-\mu, x) d x d y
\end{aligned}
$$

From equation (21), we know that the pdf of $U_{t}$ is related to the one of $S_{t}$, momentarily noted $g_{s, u, v}(t, \tau)$, as follows:

$$
g_{s, u, v}(t, \tau)=\frac{(t-u)}{\alpha \tau} p_{U}(\tau, t-u) .
$$

Then if $\tau=\mu-v$ and $t-u=y$, we have then

$$
\begin{aligned}
(\mu-v) g_{s, u, v}(u+y, \mu-v) & =\frac{y}{\alpha} p_{U}(\mu-v, y), \\
(\lambda-\mu) g_{s, u, v}(u+x, \lambda-\mu) & =\frac{x}{\alpha} p_{U}(\lambda-\mu, x) .
\end{aligned}
$$

The first term of Equation (41) is developped as follows:

$$
\begin{aligned}
& \int_{0}^{\infty} \int_{0}^{\lambda} P_{s, u, v}\left(\mu \leq S_{t_{1}}, \lambda \leq S_{t_{2}}\right) Q^{2} e^{Q(\lambda-\mu)} d \mu d \lambda \\
= & -Q^{2} \int_{\mu=0}^{\infty} \int_{\lambda=\mu}^{\infty} \int_{y=0}^{t_{1}-u} p_{U}(\mu-v, y) d y \int_{x=0}^{t_{2}-y-u} p_{U}(\lambda-\mu, x) d x d y e^{Q(\lambda-\mu)} d \lambda d \mu \\
= & Q^{2} \int_{y=0}^{t_{1}-u} \int_{x=0}^{t_{2}-y-u} \int_{\mu=v}^{\infty} p_{U}(\mu-v, y) \int_{\lambda=\mu}^{\infty} p_{U}(\lambda-\mu, x) e^{Q(\lambda-\mu)} d \lambda d \mu d x d y \\
= & Q^{2} \int_{y=0}^{t_{1}-u} \frac{\alpha}{y} \int_{x=0}^{t_{2}-y-u} \frac{\alpha}{x} \int_{\mu=v}^{\infty}(\mu-v) g_{s, u, v}(u+y, \mu-v) \\
& \int_{\lambda=\mu}^{\infty}(\lambda-\mu) g_{s, u, v}(u+x, \lambda-\mu) e^{Q(\lambda-\mu)} d \nu d \mu d x d y
\end{aligned}
$$


After simplification, we obtain that

$$
\begin{gathered}
-\int_{0}^{\infty} \int_{0}^{\lambda} P_{s, u, v}\left(\mu \leq S_{t_{1}}, \lambda \leq S_{t_{2}}\right) Q^{2} e^{Q(\lambda-\mu)} d \mu d \lambda \\
=-Q^{2} \int_{y=0}^{t_{1}-u} \frac{\alpha}{y} \int_{x=0}^{t_{2}-y-u} \frac{\alpha}{x} \int_{w=0}^{\infty} w g_{s, u, v}(u+y, w) d w \\
\int_{z=0}^{\infty} z g_{s, u, v}(u+x, z) e^{Q z} d z d x d y .
\end{gathered}
$$

Using corollary 4.3 and the relation,

$$
\begin{aligned}
\int_{w=0}^{\infty} w g_{s, u, v}(u+y, w) d w & =\mathbb{E}\left(S_{u+y}-S_{s} \mid S_{s}=v, U_{S_{s}}=u\right) \\
& =\frac{(u+y-u)^{\alpha}}{\Gamma(\alpha+1)}=\frac{y^{\alpha}}{\Gamma(\alpha+1)},
\end{aligned}
$$

leads to the following simplification of Equation (44):

$$
\begin{aligned}
-\int_{0}^{\infty} & \int_{0}^{\lambda} P_{s, u, v}\left(\mu \leq S_{t_{1}}, \lambda \leq S_{t_{2}}\right) Q^{2} e^{Q(\lambda-\mu)} d \mu d \lambda \\
= & \frac{-\alpha Q}{\Gamma(\alpha+1)} \int_{y=0}^{t_{1}-u} y^{\alpha-1} \int_{x=0}^{t_{2}-y-u} \frac{d}{d x} \boldsymbol{E}_{\alpha}\left(Q x^{\alpha}\right) d x d y \\
= & \frac{-\alpha Q}{\Gamma(\alpha+1)} \int_{y=0}^{t_{1}-u} y^{\alpha-1}\left(\boldsymbol{E}_{\alpha}\left(Q\left(t_{2}-y-u\right)^{\alpha}\right)-I\right) d y \\
= & \frac{-\alpha Q}{\Gamma(\alpha+1)} \int_{y=0}^{t_{1}-u} y^{\alpha-1} \boldsymbol{E}_{\alpha}\left(Q\left(t_{2}-y-u\right)^{\alpha}\right) d y+\frac{Q\left(t_{1}-u\right)^{\alpha}}{\Gamma(\alpha+1)} .
\end{aligned}
$$

Collecting all terms allows us to deduce Equation (39).

From the previous proposition, we can calculate the probability that the time-changed Markov chain transits from state $e_{i}$ at time $t$ to state $e_{j}$ at time $t+\Delta$, conditionally to the information available at time $s \leq u \leq t$. Let us recall that the filtration of $S_{t}$ is denoted by $\mathcal{G}_{t}$. Therefore using nested expectations and since $\theta_{S_{t}}$ is a Markov chain conditionally to $\mathcal{G}_{t}$, we deduce that:

$$
\begin{aligned}
& P\left(\theta_{S_{t+\Delta}}=e_{j} \mid \theta_{S_{t}}=e_{i}, S_{s}=v, U_{s}=u, \theta_{S_{s}}=\theta_{v}\right) \\
& \quad=\mathbb{E}_{s, u, v}\left(\mathbb{E}\left(\mathbf{1}_{\left\{\theta_{S_{t+\Delta}}=e_{j} \mid \theta_{S_{t}}=e_{i}\right\}} \mid \mathcal{G}_{t+\Delta}, U_{s}=u, \theta_{S_{s}}=\theta_{v}\right)\right) \\
& \quad=e_{i}^{\top} \mathbb{E}_{s, u, v}\left(e^{Q\left(S_{t+\Delta}-S_{t}\right)}\right) e_{j},
\end{aligned}
$$

where $\mathbb{E}_{s, u, v}\left(e^{Q\left(S_{t+\Delta}-S_{t}\right)}\right)$ is given by Equation (39). The next proposition shows that this expectation admits a limit when $\Delta \rightarrow 0$.

Corollary 4.5. The instantaneous probabilities of transition at time $t$, conditionally to $S_{s}$ and $U_{s}$ is defined as the limit

$$
\begin{aligned}
Q(t \mid s, u, v) & =\lim _{\Delta \rightarrow 0} \frac{\mathbb{E}_{s, u, v}\left(e^{Q\left(S_{t+\Delta}-S_{t}\right)}\right)-I}{\Delta} \\
& =\frac{\alpha Q(t-u)^{\alpha-1}}{\Gamma(\alpha+1)} .
\end{aligned}
$$

Proof From Proposition 4.4 in which we set $t_{1}=t_{2}$, we infer that the identity matrix is equal to the following sum

$$
\begin{aligned}
I= & \frac{-\alpha Q}{\Gamma(\alpha+1)} \int_{0}^{t-u} y^{\alpha-1} \boldsymbol{E}_{\alpha}\left(Q(t-y-u)^{\alpha}\right) d y \\
& +\boldsymbol{E}_{\alpha}\left(Q(t-u)^{\alpha}\right)
\end{aligned}
$$


Injecting this expression in Eq. (46) and passing to the limit gives us

$$
\begin{aligned}
\lim _{\Delta \rightarrow 0} \frac{\mathbb{E}_{s, u, v}\left(e^{Q\left(S_{t+\Delta}-S_{t}\right)}\right)-I}{\Delta}= & \left.\frac{-\alpha Q}{\Gamma(\alpha+1)} \int_{y=0}^{t-u} y^{\alpha-1} \frac{\partial \boldsymbol{E}_{\alpha}\left(Q(x)^{\alpha}\right)}{\partial x}\right|_{x=t-y-u} d y \\
& +\left.\frac{\partial \boldsymbol{E}_{\alpha}\left(Q(x)^{\alpha}\right)}{\partial x}\right|_{x=t-u}
\end{aligned}
$$

By definition, $\frac{\partial \boldsymbol{E}_{\alpha}\left(Q(x)^{\alpha}\right)}{\partial x}=\sum_{n=1}^{\infty} \frac{\alpha n Q^{n} x^{\alpha n-1}}{\Gamma(n \alpha+1)}$. The integral in Eq. (47) after the change of variable $y=(t-u) z$ becomes

$$
\begin{aligned}
\int_{y=0}^{t-u} & \left.y^{\alpha-1} \frac{\partial \boldsymbol{E}_{\alpha}\left(Q(x)^{\alpha}\right)}{\partial x}\right|_{x=t-y-u} d y \\
& =\sum_{n=1}^{\infty} \frac{\alpha n Q^{n}}{\Gamma(n \alpha+1)} \int_{y=0}^{t-u} y^{\alpha-1}(t-u-y)^{n \alpha-1} d y \\
& =\sum_{n=1}^{\infty} \frac{\alpha n Q^{n}}{\Gamma(n \alpha+1)}(t-u)^{(n+1) \alpha-1} \int_{0}^{1} z^{\alpha-1}(1-z)^{n \alpha-1} d z \\
& =\sum_{n=1}^{\infty} \frac{Q^{n}}{\Gamma(n \alpha)}(t-u)^{(n+1) \alpha-1} B(\alpha, n \alpha) .
\end{aligned}
$$

where $B(\alpha, n \alpha)$ is the beta function. Since $B(x, y)=\frac{\Gamma(x) \Gamma(y)}{\Gamma(x+y)}$, this last expression is rewritten as follows:

$$
\begin{gathered}
\left.\int_{0}^{t-u} y^{\alpha-1} \frac{\partial \boldsymbol{E}_{\alpha}\left(Q(x)^{\alpha}\right)}{\partial x}\right|_{x=t-y-u} d y \\
=\sum_{n=1}^{\infty} \frac{\Gamma(\alpha) Q^{n}(t-u)^{(n+1) \alpha-1}}{\Gamma((n+1) \alpha)} .
\end{gathered}
$$

The first term in Equation (47) is then equal to the following sum

$$
\begin{gathered}
\left.\frac{-\alpha Q}{\Gamma(\alpha+1)} Q \int_{0}^{t-u} y^{\alpha-1} \frac{\partial \boldsymbol{E}_{\alpha}\left(Q(x)^{\alpha}\right)}{\partial x}\right|_{x=t-y-u} d y \\
=-\frac{1}{(t-u)} \sum_{n=1}^{\infty} \frac{Q^{n+1}(t-u)^{(n+1) \alpha}}{\Gamma((n+1) \alpha)} \\
=-\frac{1}{(t-u)} \sum_{n=2}^{\infty} \frac{\alpha n Q^{n}(t-u)^{\alpha n}}{\Gamma(n \alpha+1)} .
\end{gathered}
$$

Given that the derivative of the Mittag-Leffler function is

$$
\begin{aligned}
\left.\frac{\partial \boldsymbol{E}_{\alpha}\left(Q(x)^{\alpha}\right)}{\partial x}\right|_{x=t-u} & =\sum_{n=1}^{\infty} \frac{\alpha n Q^{n}(t-u)^{\alpha n-1}}{\Gamma(n \alpha+1)} \\
& =\frac{1}{(t-u)} \sum_{n=1}^{\infty} \frac{\alpha n Q^{n}(t-u)^{\alpha n}}{\Gamma(n \alpha+1)},
\end{aligned}
$$

we infer Equation (46).

If we set $\alpha=1$, Equation (46) becomes $Q(t \mid s, u, v)=Q$ that is well the instantaneous probability of transition of the non-time changed Markov chain. 


\section{Actuarial valuation with the fractional chain}

The next proposition presents the conditional expectation of integral over $[t, T]$ of the discounted chain vector. This result is required to calculate the value of future discounted premiums and annuities.

Proposition 5.1. For $0 \leq t \leq u \leq T, v \geq 0$, and if $\Gamma(t, \omega)=\int_{0}^{t} e^{-z} z^{\omega-1} d z$ is the lower incomplete Gamma, we have the following equality

$$
\begin{gathered}
\mathbb{E}_{t, u, v}\left(\int_{t}^{T} e^{-r(x-t)} \theta_{S_{x}}^{\top} d x\right)=\frac{\theta_{v}^{\top}}{r}\left(1-e^{-r(u-t)}\right) \\
+e^{-r(u-t)} \theta_{v}^{\top} \sum_{n=0}^{\infty} \frac{Q^{n} \Gamma(r(T-u), n \alpha+1)}{\Gamma(n \alpha+1) r^{n \alpha+1}} .
\end{gathered}
$$

If $T=\infty$, at inception of the contract $(t=0, v=0$ and $u=0)$, this expectation becomes

$$
\mathbb{E}_{0}\left(\int_{0}^{T} e^{-r(x-t)} \theta_{S_{x}}^{\top} d x\right)=\frac{\theta_{0}^{\top}}{r}\left(I-Q r^{-\alpha}\right)^{-1} .
$$

Proof Using the Fubini's theorem, the expectation (48) is the integral of expectations, and from (33) we obtain that

$$
\begin{gathered}
\mathbb{E}_{t, u, v}\left(\int_{t}^{T} e^{-r(x-t)} \theta_{S_{x}}^{\top} d x\right)=\int_{t}^{u} e^{-r(x-t)} \mathbb{E}_{t, u, v}\left(\theta_{S_{x}}^{\top}\right) d x+\int_{u}^{T} e^{-r(x-t)} \mathbb{E}_{t, u, v}\left(\theta_{S_{x}}^{\top}\right) d x \\
=\theta_{v}^{\top} \int_{t}^{u} e^{-r(x-t)} I d x+e^{-r(u-t)} \theta_{v}^{\top} \int_{u}^{T} e^{-r(x-u)} \boldsymbol{E}_{\alpha}\left(Q(x-u)^{\alpha}\right) d x \\
=\frac{\theta_{v}^{\top}}{r}\left(1-e^{-r(u-t)}\right)+e^{-r(u-t)} \theta_{v}^{\top} \int_{u}^{T} e^{-r(x-u)} \boldsymbol{E}_{\alpha}\left(Q(x-u)^{\alpha}\right) d x
\end{gathered}
$$

We replace the Mittag-Leffler function by its definition and rewrite the integral in this last equation as an infinite sum

$$
\int_{u}^{T} e^{-r(x-u)} \boldsymbol{E}_{\alpha}\left(Q(x-u)^{\alpha}\right) d x=\sum_{n=0}^{\infty} \frac{Q^{n}}{\Gamma(n \alpha+1)} \int_{u}^{T} e^{-r(x-u)}(x-u)^{n \alpha} d x .
$$

Performing the change of variable $z=r(x-u)$ and by definition of the lower incomplete Gamma function, the integral in this sum is rewritten as

$$
\begin{aligned}
\int_{u}^{T} e^{-r(x-u)}(x-u)^{n \alpha} d x & =\frac{1}{r^{n \alpha+1}} \int_{0}^{r(T-u)} e^{-z} z^{(n \alpha+1)-1} d z \\
& =\frac{\Gamma(r(T-u), n \alpha+1)}{r^{n \alpha+1}},
\end{aligned}
$$

which allows to obtain Equation (48). At inception, $t=0$ and $u=0$. Under the assumption that $T=\infty$, the integral of discounted matrix Mittag-Leffler function becomes an infinite sum

$$
\begin{aligned}
\lim _{T \rightarrow \infty} \int_{0}^{T} e^{-r x} \boldsymbol{E}_{\alpha}\left(Q x^{\alpha}\right) d x & =\sum_{n=0}^{\infty} \frac{Q^{n}}{\Gamma(n \alpha+1) r^{n \alpha+1}} \int_{0}^{\infty} e^{-z} z^{(n \alpha+1)-1} d z \\
& =\frac{1}{r} \sum_{n=0}^{\infty}\left(Q r^{-\alpha}\right)^{n}
\end{aligned}
$$

As all eigenvalues of $I-Q r^{-\alpha}$ are strictly different from zero, its inverse , $\left(I-Q r^{-\alpha}\right)^{-1}$ exists. In order to conclude, we have to show that $\left(I-Q r^{-\alpha}\right)^{-1}$ admits a representation as an infinite serie 
$\sum_{n=0}^{\infty}\left(Q r^{-\alpha}\right)^{n}$. This may be proven by showing that the product of $I-Q r^{-\alpha}$ times $\sum_{n=0}^{\infty}\left(Q r^{-\alpha}\right)^{n}$ is equal to the identity matrix as follows:

$$
\begin{aligned}
(I & \left.-Q r^{-\alpha}\right) \sum_{n=0}^{\infty}\left(Q r^{-\alpha}\right)^{n} \\
& =\sum_{n=0}^{\infty}\left(Q r^{-\alpha}\right)^{n}-\sum_{n=0}^{\infty}\left(Q r^{-\alpha}\right)^{n}+I \\
& =I .
\end{aligned}
$$

We deduce therefore that

$$
\int_{0}^{\infty} e^{-r x} \boldsymbol{E}_{\alpha}\left(Q x^{\alpha}\right) d x=\frac{1}{r}\left(I-Q r^{-\alpha}\right)^{-1}
$$

and obtain Equation (49).

In order to estimate the present value of benefits paid at transitions of the Markov chain, we evaluate the expectation of $\mathbf{1}_{\left\{\theta_{S_{t}}=e_{i}\right\}} \frac{d N_{S_{t}}^{i, j}}{d t}$. We denote by $\delta_{i, j}=0$ if $i \neq j$ and $\delta_{i, j}=1$ if $i=j$. Conditionally to the filtration $\mathcal{G}_{t}$ of $S_{t}, \theta_{S_{t}}$ is a Markov chain. For $s \leq u \leq t$, we nest expectations in order to obtain that for all $i, j=1, \ldots, l$

$$
\begin{aligned}
& \mathbb{E}_{s, u, v}\left(\mathbf{1}_{\left\{\theta_{S_{t}}=e_{i}\right\}} \frac{d N_{S_{t}}^{i, j}}{d t}\right) \\
& =\mathbb{E}_{s, u, v}\left(\mathbb{E}_{s, u, v}\left(\mathbf{1}_{\left\{\theta_{S_{t}}=e_{i}\right\}} \lim _{\Delta \rightarrow 0}\left(\frac{\mathbf{1}_{\left\{\theta_{S_{t+\Delta}}=e_{j} \mid \theta_{S_{t}}=e_{i}\right\}}-\delta_{i, j}}{\Delta}\right) \mid \mathcal{G}_{t+\Delta}\right)\right) \\
& =\mathbb{E}_{s, u, v}\left(P\left(\theta_{S_{t}}=e_{i} \mid \theta_{S_{s}}=\theta_{v}, \mathcal{G}_{t+\Delta}\right) \lim _{\Delta \rightarrow 0}\left(\frac{P\left(\theta_{S_{t+\Delta}}=e_{j} \mid \theta_{S_{t}}=e_{i}, \mathcal{G}_{t+\Delta}\right)-\delta_{i, j}}{\Delta}\right)\right) \\
& =\mathbb{E}_{s, u, v}\left(\theta_{v}^{\top} e^{Q\left(S_{t}-S_{s}\right)} e_{i} e_{i}^{\top} \lim _{\Delta \rightarrow 0}\left(\frac{e^{Q\left(S_{t+\Delta}-S_{t}\right)}-I}{\Delta}\right) e_{j}\right) .
\end{aligned}
$$

The sum of this expectation multiplied by benefits with respect to $i=1, \ldots, l$ is then rewritten in a matrix form

$$
\begin{aligned}
& \sum_{i=1}^{l} \mathbb{E}_{s, u, v}\left(d_{i, j} \mathbf{1}_{\left\{\theta_{S_{t}}=e_{i}\right\}} \frac{d N_{S_{t}}^{i, j}}{d t}\right) \\
& =\theta_{v}^{\top} \mathbb{E}_{s, u, v}\left(e^{Q\left(S_{t}-S_{s}\right)} \lim _{\Delta \rightarrow 0}\left(\frac{e^{Q\left(S_{t+\Delta}-S_{t}\right)}-I}{\Delta}\right)\right) \otimes D e_{j} \\
& =\theta_{v}^{\top}\left(\lim _{\Delta \rightarrow 0} \mathbb{E}_{s, u, v}\left(\frac{e^{Q\left(S_{t+\Delta}-S_{s}\right)}-e^{Q\left(S_{t}-S_{s}\right)}}{\Delta}\right)\right) \otimes D e_{j}
\end{aligned}
$$

For $s \leq t \leq u$, the chain is motionless and therefore $\mathbb{E}_{s, u, v}\left(\mathbf{1}_{\left\{\theta_{S_{t}}=e_{i}\right\}} \frac{d N_{S_{t}}^{i, j}}{d t}\right)=0$. The following proposition shows that the limit in Equation (50) is well defined.

Proposition 5.2. For $0 \leq s \leq u \leq t$ and $v \geq 0$, the expectation of the limit in Equation (50) is equal to

$$
\mathbb{E}_{s, u, v}\left(\lim _{\Delta \rightarrow 0} \frac{\left(e^{Q\left(S_{t+\Delta}-S_{s}\right)}-e^{Q\left(S_{t}-S_{s}\right)}\right)}{\Delta}\right)=\sum_{n=1}^{\infty} \frac{Q^{n}(t-u)^{n \alpha-1}}{\Gamma(n \alpha)}
$$

For $0 \leq s \leq t \leq u$, this limit is null. 
From Corollary 3.3, this expectation is

$$
\begin{aligned}
& \mathbb{E}_{s, u, v}\left(\lim _{\Delta \rightarrow 0} \frac{\left(e^{Q\left(S_{t+\Delta}-S_{s}\right)}-e^{Q\left(S_{t}-S_{s}\right)}\right)}{\Delta}\right) \\
& =\lim _{\Delta \rightarrow 0} \frac{\boldsymbol{E}_{\alpha}\left(Q(t+\Delta-u)^{\alpha}\right)-\boldsymbol{E}_{\alpha}\left(Q(t-u)^{\alpha}\right)}{\Delta} \\
& =\left.\frac{\partial \boldsymbol{E}_{\alpha}\left(Q x^{\alpha}\right)}{\partial x}\right|_{x=t-u} .
\end{aligned}
$$

This derivative is equal to Equation (51).

Proposition 5.3. For $0 \leq t \leq u \leq T$ and $v>0$, the expected sum of discounted benefits, conditionally to $S_{t}$ and $U_{S_{t}}$, is given by

$$
\begin{gathered}
\sum_{i=1}^{l} \sum_{j=1, i \neq j}^{l} \mathbb{E}_{t, u, v}\left(\int_{t}^{T} e^{-r(x-t)} \mathbf{1}_{\left\{\theta_{S_{x}}=e_{i}\right\}} d_{i, j} d N_{S_{x}}^{i, j}\right) \\
=\theta_{v}^{\top} e^{-r(u-t)} \sum_{n=1}^{\infty} \frac{Q^{n} \Gamma(r(T-u), n \alpha)}{r^{n \alpha} \Gamma(n \alpha)} \otimes D \boldsymbol{e}
\end{gathered}
$$

where $\Gamma(x, y)$ is the lower Gamma incomplete function. If $T=\infty$, at inception of the contract $(t=0, v=0$ and $u=0)$, we have

$$
\mathbb{E}_{0}\left(\int_{0}^{\infty} e^{-r x} \mathbf{1}_{\left\{\theta_{S_{x}}=e_{i}\right\}} d_{i, j} d N_{S_{x}}^{i, j}\right)=\frac{\theta_{0}^{\top}}{r^{\alpha}}\left(I-Q r^{-\alpha}\right)^{-1} Q \otimes D \boldsymbol{e}
$$

Proof From Proposition 5.2, the sum of discounted expected benefits paid at transitions is rewritten as an infinite sum

$$
\begin{aligned}
& \sum_{j=1}^{l} \mathbb{E}_{t, u, v}\left(\int_{t}^{T} e^{-r(x-t)} \mathbf{1}_{\left\{\theta_{S_{x}}=e_{i}\right\}} d_{i, j} d N_{S_{x}}^{i, j}\right) \\
& \quad=\sum_{j=1}^{l} \theta_{v}^{\top} \sum_{n=1}^{\infty} \frac{Q^{n}}{\Gamma(n \alpha)} \int_{u}^{T} e^{-r(x-t)}(x-u)^{n \alpha-1} d x \otimes D e_{j}
\end{aligned}
$$

If we perform the change of variable, $z=r(x-u)$, the integrals in this last equation may be rewritten as incomplete lower Gamma functions:

$$
\begin{aligned}
& \int_{u}^{T} e^{-r(x-t)}(x-u)^{n \alpha-1} d x=\frac{e^{-r(u-t)}}{r^{n \alpha}} \int_{0}^{r(T-u)} e^{-z} z^{n \alpha-1} d z \\
& =\frac{e^{-r(u-t)}}{r^{n \alpha}} \Gamma(r(T-u), n \alpha) .
\end{aligned}
$$

Combining Equations (54) and (55) leads to the expression (52). If $T=\infty, t=u=0$ then $\int_{0}^{\infty} e^{-z} z^{n \alpha-1} d z=\Gamma(n \alpha)$ and we infer that

$$
\sum_{j=1}^{l} \mathbb{E}_{0}\left(\int_{0}^{\infty} e^{-r x} \mathbf{1}_{\left\{\theta_{S_{x}}=e_{i}\right\}} d_{i, j} d N_{S_{x}}^{i, j}\right)=\sum_{j=1}^{l} \theta_{0}^{\top} \sum_{n=1}^{\infty} \frac{Q^{n}}{r^{n \alpha}} \otimes D e_{j} .
$$

Using a similar reasoning to the proof of Proposition 5.1, we deduce that

$$
\begin{aligned}
\sum_{n=1}^{\infty} \frac{Q^{n}}{r^{n \alpha}} & =\frac{1}{r^{\alpha}}\left(\sum_{n=0}^{\infty}\left(Q r^{-\alpha}\right)^{n}\right) Q \\
& =\frac{1}{r^{\alpha}}\left(I-Q r^{-\alpha}\right)^{-1} Q
\end{aligned}
$$


which concludes the proof.

From Propositions 5.1 and 5.3, the provision at time $t$ conditionally to $S_{t}=v, U_{S_{t}}=u$ and $\theta_{S_{t}}=\theta_{v}$, denoted by $V_{t, u, v}$, is given by the following expression

$$
\begin{aligned}
V_{t, u, v}= & e^{-r(u-t)} \theta_{v}^{\top} \sum_{n=0}^{\infty} Q^{n} \frac{\Gamma(r(T-u), n \alpha+1)}{\Gamma(n \alpha+1) r^{n \alpha+1}}(\boldsymbol{a}-\boldsymbol{c}) \\
& +e^{-r(u-t)} \theta_{v}^{\top} \sum_{n=1}^{\infty} Q^{n} \frac{\Gamma(r(T-u), n \alpha)}{\Gamma(n \alpha) r^{n \alpha}} \otimes D \boldsymbol{e} \\
& +\frac{\theta_{v}^{\top}}{r}\left(1-e^{-r(u-t)}\right)(\boldsymbol{a}-\boldsymbol{c}) .
\end{aligned}
$$

The third term on the right hand side of this equation accounts benefits and premiums paid or collected when the stochastic clock is stopped.

At inception of the contract, $U_{0}=S_{0}=0$, the fair premium rates that ensure the actuarial equilibrium between benefits and premiums, satisfy the relation

$$
0=\theta_{0}^{\top} \sum_{n=0}^{\infty} Q^{n} \frac{\Gamma(r T, n \alpha+1)}{\Gamma(n \alpha+1) r^{n \alpha+1}}(\boldsymbol{a}-\boldsymbol{c})+\theta_{0}^{\top} \sum_{n=1}^{\infty} Q^{n} \frac{\Gamma(r T, n \alpha)}{\Gamma(n \alpha) r^{n \alpha}} \otimes D \boldsymbol{e} .
$$

One natural possible solution is given by

$$
\boldsymbol{c}=\boldsymbol{a}+\left(\sum_{n=0}^{\infty} Q^{n} \frac{\Gamma(r T, n \alpha+1)}{\Gamma(n \alpha+1) r^{n \alpha+1}}\right)^{-1}\left(\sum_{n=1}^{\infty} Q^{n} \frac{\Gamma(r T, n \alpha)}{\Gamma(n \alpha) r^{n \alpha}}\right) \otimes D \boldsymbol{e} .
$$

If premiums are exclusively paid when the chain is in the initial state, the premium rate, noted $c_{0}$, is given by

$$
c_{0}=\frac{\theta_{0}^{\top}\left(\sum_{n=0}^{\infty} Q^{n} \frac{\Gamma(r T, n \alpha+1)}{\Gamma(n \alpha+1) r^{n \alpha+1}}\right) \boldsymbol{a}+\theta_{0}^{\top}\left(\sum_{n=1}^{\infty} Q^{n} \frac{\Gamma(r T, n \alpha)}{\Gamma(n \alpha) r^{n \alpha}}\right) \otimes D \boldsymbol{e}}{\theta_{0}^{\top}\left(\sum_{n=0}^{\infty} Q^{n} \frac{\Gamma(r T, n \alpha+1)}{\Gamma(n \alpha+1) r^{n \alpha+1}}\right) \theta_{0}} .
$$

Of course, the model is much more flexible and allows for various schemes of premiums payment. if $\boldsymbol{c}=\left(c_{1}, \ldots, c_{l}\right)$ where $c_{k} \neq 0$ for $k=1, \ldots, l$, the fair premium vector solves Equation (57). In this case, the solution is not unique and we must add a constraint on $\boldsymbol{c}$ in order to ensure identifiability. For instance, we can impose that

$$
c=c_{b} \times\left(\beta_{1}, \ldots, \beta_{l}\right),
$$

where $c_{b} \in \mathbb{R}^{+}$is a baseline premium rate that is modulated by a priori coefficients $\left(\beta_{k}\right)_{k=1, \ldots . l}$, depending on the insured's state.

When $T=\infty$, the fair premium rates that guarantee the actuarial equilibrium between benefits and premiums are solutions of the equation

$$
\theta_{0}^{\top}\left(r I-Q r^{1-\alpha}\right)^{-1} \boldsymbol{c}=\theta_{0}^{\top}\left(r I-Q r^{1-\alpha}\right)^{-1} \boldsymbol{a}+\theta_{0}^{\top}\left(r^{\alpha} I-Q\right)^{-1} Q \otimes D \boldsymbol{e} .
$$

A comparison with Equation (8) clearly emphasizes the impact of the time change on the valuation equation. One natural solution of Equation (60) is

$$
\boldsymbol{c}=\boldsymbol{a}+r^{1-\alpha} Q \otimes D e_{j} .
$$

This premium rate is similar to the one obtained in the non-fractional model, excepted that the third term is adjusted by a factor $r^{1-\alpha}$. If premiums are exclusively paid when the chain is in the initial state, the premium rate, noted $c_{0}$, is given by

$$
c_{0}=\frac{\theta_{0}^{\top}\left(r I-Q r^{1-\alpha}\right)^{-1} \boldsymbol{a}+\theta_{0}^{\top}\left(r^{\alpha} I-Q\right)^{-1} Q \otimes D \boldsymbol{e}}{\theta_{0}^{\top}\left(r I-Q r^{1-\alpha}\right)^{-1} \theta_{0}} .
$$


If we consider a contract that remains in force till the insured's death, the decease of the insured is modelled by an absorbing state. In this case, the transition matrix has the block structure (10) and the survival probability is provided by Equation (37). One possible solution of Equation (60) is in this scenario

$$
\boldsymbol{c}=\boldsymbol{a}+r^{1-\alpha}\left(\begin{array}{cc}
0 & \mathbf{0} \\
\boldsymbol{b} \otimes \boldsymbol{d} & B \otimes \bar{D}
\end{array}\right) \boldsymbol{e} .
$$

When processes $\left(U_{t}\right)_{t \geq 0}$ and $\left(S_{t}\right)_{t \geq 0}$ are not observed, the provision is computed by simulations for instance with the method proposed by Chambers et al. (1976). In comparison to a full MonteCarlo method, this approach avoids to perform time consuming simulations in simulations. This point is illustrated in the next section.

To close this section, we briefly discuss the estimation of the fractional Markov chain. As the chain is ruled by a hidden stochastic clock, obtaining accurate parameter estimates is a challenging task, unless we dispose of an educated guess about the matrix of transition probabilities, $P(t \mid 0,0,0)$, for different times horizons $t \in\left\{t_{1}, \ldots t_{m}\right\}$. In this case, the calibration is trivial thanks to Equation (33). Let us define the matrix

$$
A(t \mid Q, \alpha)=P(t \mid 0,0,0)-\boldsymbol{E}_{\alpha}\left(Q t^{\alpha}\right),
$$

of spreads between modeled and observed transition probabilities. The estimates $\hat{Q}, \hat{\alpha}$ of $Q$ and $\alpha$ are obtained by least square minimization as follows:

$$
\hat{Q}, \hat{\alpha}=\arg \min _{Q, \alpha} \sum_{k=1}^{m} \operatorname{tr}\left(A(t \mid Q, \alpha)^{\top} A(t \mid Q, \alpha)\right)^{\top} \boldsymbol{e},
$$

where $\boldsymbol{e}$ is a $l$-vector of ones. We can eventually weight the matrix of spreads in function of the reliability of guessed transition probabilities.

\section{Numerical illustration}

We consider that the health status of an individual is modeled by a Markov chain with three states. The first one is absorbing and reached when the insured dies. The second state corresponds to disability whereas the third regime is in force at the inception of the contract. Premiums are exclusively paid in this third regime. When the insured deceases, the insurance policy pays a lump sum of 10 monetary units. During a disability period, the insured receives a continuous annuity rate of 1 monetary unit. The matrix of one-year transition probabilities (before time-change) and the corresponding matrix $Q$ are set to:

$$
P(0,1)=\left(\begin{array}{ccc}
1.0 & 0.0 & 0.0 \\
0.2 & 0.8 & 0.0 \\
0.05 & 0.05 & 0.9
\end{array}\right) \quad Q=\left(\begin{array}{ccc}
0.0 & 0.0 & 0.0 \\
0.2231 & -0.2231 & 0.0 \\
0.0465 & 0.0589 & -0.1054
\end{array}\right)
$$

The matrix $D$ and vector $a$ are in this case given by:

$$
D=\left(\begin{array}{ccc}
0 & 0 & 0 \\
10 & 0 & 0 \\
10 & 0 & 0
\end{array}\right) \quad a=\left(\begin{array}{l}
0 \\
1 \\
0
\end{array}\right)
$$

We use Equation (56) to calculate the premium rate of this contract for maturities ranging from 1 to 20 years. We impose that $\boldsymbol{c}=\left(c_{0}, 0,0\right)^{\top}$ in order to take into account that premiums are only paid when the chain is in the third state. The risk-free rate is set to $r=5 \%$. The left plot of Figure 2 shows the premium rates for maturities that range from 1 to 20 years and for various levels of $\alpha$. For most of maturities, we observe that the lower is $\alpha$, the lower is the premium rate. This may be explained by the fact that the chain stays further in the same regime in a fractional setting than in the corresponding Markov chain. This point is emphasized by the right plot of Figure 2 presenting the survival probabilities for different time horizons and when the individual is 


\begin{tabular}{|c|cccc|c|}
\hline$\alpha$ & $T=1$ & $T=5$ & $T=10$ & $T=20$ & $T=\infty$ \\
\hline 0.5 & 0.673 & 0.407 & 0.353 & 0.322 & 0.302 \\
0.7 & 0.638 & 0.544 & 0.533 & 0.526 & 0.514 \\
0.9 & 0.585 & 0.714 & 0.805 & 0.868 & 0.882 \\
1.0 & 0.554 & 0.813 & 0.991 & 1.122 & 1.161 \\
\hline
\end{tabular}

Table 1: Premium rates for different time horizons and values of $\alpha$.

alpha $=0.9$

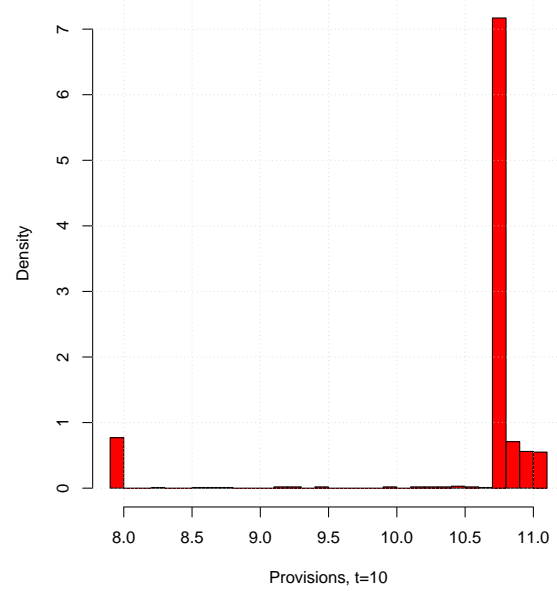

alpha $=0.5$

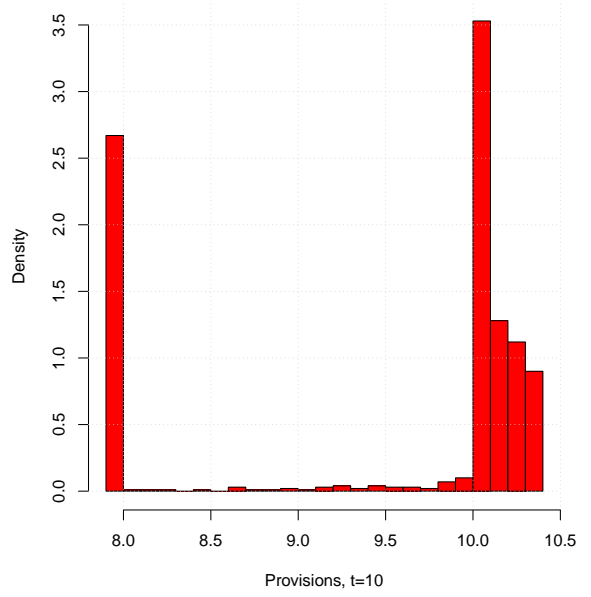

Figure 3: Histograms of provisions at time $t=10$ if $\alpha=0.9$ and $\alpha=0.5$.

initially in state 3 . This graph clearly reveals that survival probabilities are inversely proportional to $\alpha$.
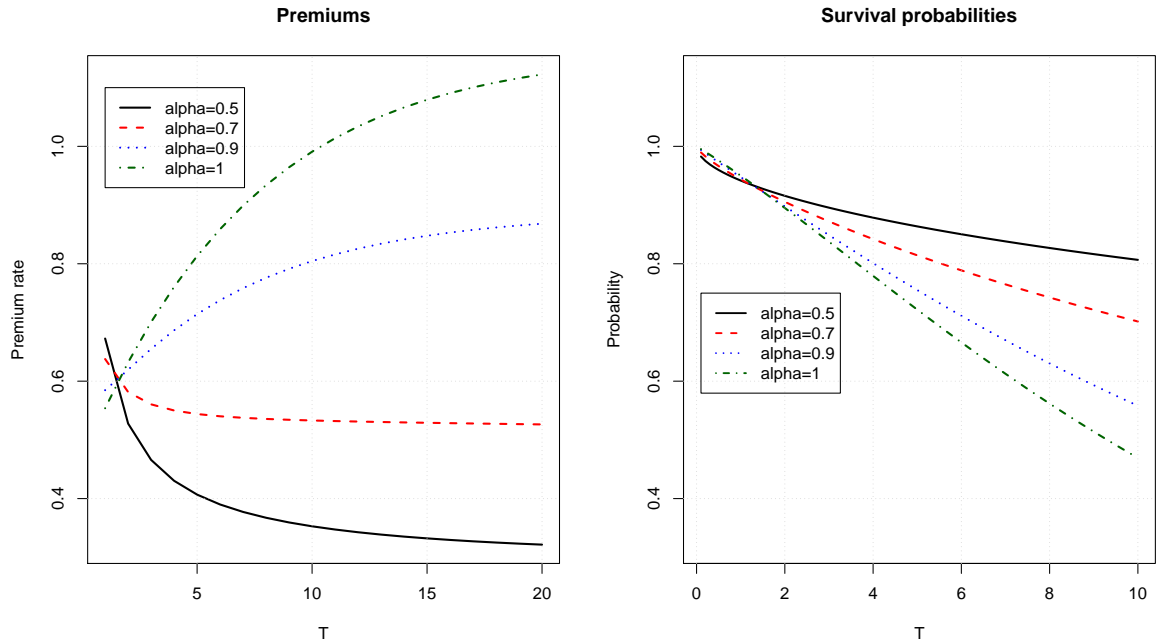

Figure 2: Left plot: premium rates for $T=1$ to 20 years. Right plot : survival probabilities.

Table 1 reports some premium rates presented in the left plot of Figure 2 and compare them to the asymptotic premium when $T \rightarrow \infty$. This underlines the convergence of premiums to these asymptotic values for policies with an initial maturity longer than 15 years.

In the previous section, we have found a closed form expression for provisions, conditionally to $S_{t}$ and $U_{S_{t}}$. In practice, processes $\left(U_{t}\right)_{t>0}$ and $\left(S_{t}\right)_{t>0}$ are not observable but they can be simulated with the method proposed by Chambers et al. (1976), e.g. implemented in the R package 


\begin{tabular}{|ccccc|}
\hline$\alpha$ & Mean & Standard & \multicolumn{2}{c|}{ Percentiles } \\
& $V_{t=10}$ & Deviation & $5 \%$ & $95 \%$ \\
\hline 1.00 & 11.06 & - & - & - \\
0.90 & 10.497 & 0.827 & 7.911 & 11.027 \\
0.70 & 9.503 & 0.976 & 7.965 & 10.323 \\
0.50 & 8.818 & 0.741 & 8.079 & 9.718 \\
\hline
\end{tabular}

Table 2: Provisions at time $t=10$ for different levels of $\alpha$.

\begin{abstract}
"stabledist". We perform 1000 simulations and estimate the provisions of a 20 years contract at time $t=10$ with Equation (56). The left and right plots of Figure 3 respectively show the empirical probability density functions of these provisions for $\alpha=0.9$ and $\alpha=0.5$, when the individual is in the disability regime. This density is bimodal and exhibits a first mode around $7.89=\frac{1}{r}\left(1-e^{-r 10}\right)$, that is the cost of a continuous annuity for a period of 10 years. This peak is directly related to simulations in which $U_{S_{10}} \geq 20$. In these scenarios, the clock $S_{t}$ is stopped between $t=10$ and $t=20$ years. The chain being frozen in the disability regime during this period, the insurer's liability is a pure financial annuity. Table 2 reports the means, standard deviations and percentiles of the distributions of provisions. The provisions are on average lower in the fractional case than in the Markov setting. Again, this is explainable by the higher survival probabilities yield by fractional models.
\end{abstract}

\title{
7 Conclusions
}

This article explores the features of a fractional multi-states model and its applications to insurance. This fractional process is built as a Markov chain that is subordinated by the inverse of an $\alpha$ stable process. Compared to a classical Markov framework, the sojourn time in a state is distributed according to a Mittag-Leffler probability law that is a sub-exponential distribution. We show that the matrix of transition probabilities is solution of a fractional Kolmogorov differential equation and that instantaneous probabilities are time-dependent, as for a non-homogeneous Markov chain.

Results obtained in this article rely on original extensions of well-known properties of an inverse $\alpha$-stable process conditioned by its filtration at birth. Conditionally to information about the time-change and the inverted $\alpha$-stable processes, most of them can be adapted up to later times. This allows us not only to price insurance policies but also to evaluate their provisions at any times before expiry with closed-form expressions. As illustrated in the last section, in absence of information about the stochastic clock, our results can be combined with Monte-Carlo simulations in order to estimate the distribution of provisions. We observe that under certain circumstances, this distribution is bimodal due to periods during which the chain is motionless.

\section{Acknowledgment}

This work was supported by the "Fonds de la Recherche Scientifique" - FNRS under Grant n ${ }^{\circ}$ 33658713.

\section{References}

[1] Albrecher H., Bladt M., Bladt M. 2020. Multivariate fractional phase-type distributions. Fractional Calculus and Applied Analysis. In press.

[2] Aletti G., Leonenko N.N., Merzbach E. 2018. Fractional Poisson processes and martingales. J. Stat. Phys. 170 (4), 700-730. 
[3] Applebaum 2009. Lévy processes and stochastic calculus. Second Edition. Cambridge University Press.

[4] Beghin L., Orsingher E. 2009. Fractional Poisson processes and related planar random motions. Electron. J. Probab. 14(61), 1790-1827.

[5] Biard R., Saussereau B. 2018. Fractional Poisson process: long-range dependence and applications in ruin theory. Journal of Applied Probability, 51 (3), 727 - 740.

[6] Brociek R., Slota D., Krol M., Matula G., Kwasny W., 2017. Modeling of heat distribution in porous aluminum using fractional differential equation. Fractal and Fractional 1(1), 1-9.

[7] Chambers J.M., Mallows C.L., Stuck, B.W. 1976. A method for simulating stable random variables. Journal of American Statistical Association, 71, 340-344.

[8] Denuit M., Robert C., 2007. Actuariat des assurances de personnes. Modélisation, tarification et provisionnement. Economica Paris.

[9] D'Amico G., Guillen M., Manca R., 2009. Full backward non-homogeneous semi-Markov processes for disability insurance models: A Catalunya real data application. Insurance: Mathematics \& Economics. 45 (2) 173-179.

[10] Hainaut D. 2020. Fractional Hawkes processes. Physica A: Statistical Mechanics and its Applications, 549, 124330.

[11] Hainaut D., Leonenko N. 2021. Option pricing in illiquid markets: a fractional jump-diffusion approach. Journal of Computational and Applied Mathematics. 381 (1), 112995.

[12] Kumar A., Leonenko N., Pichler A. 2020. Fractional risk process in insurance. Mathematics and Financial Economics, 14, 43-65.

[13] Janssen J., De Dominicis R. 1984. Finite non-homogeneous semi-Markov processes. Insurance: Mathematics and Economics, 3, 157-165.

[14] Janssen J., Manca R. 1997. A realistic non-homogeneous stochastic pension funds model on scenario basis. Scandinavian Actuarial Journal, 113-137

[15] Leonenko N., Meerschaert M., Sikorskii A. 2013. Correlation structure of fractional Pearson diffusions. Computers and Mathematics with Applications, 66, 737-745.

[16] Magdziarz M. 2009. Black-Scholes formula in subdiffusive regime. Journal of Statistical Physics, 136, 553-564.

[17] Meerschaert M., Straka P., 2013. Inverse Stable Subordinators. Math. Model. Nat. Phenom. $8(2), 1-16$.

[18] Musson R. M. W., Tsapanos T., Nakas C. T. 2002. A power-law function for earthquake interarrival time and magnitude, Bulletin of the Seismological Society of America, 92 (5), $1783-1794$

[19] Norberg R. 2002. Basic life insurance mathematics. Lecture notes, Laboratory of Actuarial Mathematics, University of Copenhagen.

[20] Piryatinska A., Saichev A. I., Woyczynski W. A. 2005. Models of anomalous diffusion: the subdiffusive case. Physica A: Statistical Mechanics and its Applications, 349 (3), 375-420.

[21] Rolski T., Schmidli H., Schmidt V., Teugels J. 1999. Stochastic processes for insurance and finance. Wiley - Chichester.

[22] Zadeh A.H., Jones B.L., Stanford D.A. 2014. The use of phase-type models for disability insurance calculations. Scandinavian Actuarial Journal, 2014 (8), 714-728. 